\title{
Therapeutic tissue regenerative nanohybrids self-assembled from bioactive inorganic core / chitosan shell nanounits
}

\author{
Han-Sem Kim ${ }^{\S, 1,2}$, Jung-Hwan Lee ${ }^{\S, 1,2,3,4,5}$, Nandin Mandakhbayar ${ }^{\S, 1,2}$, Guang-Zhen Jin ${ }^{1,2,4}$, \\ Sung-Jin Kim ${ }^{1,2}$, Ji-Young Yoon ${ }^{1,2}$, Seung Bin Jo ${ }^{1,2}$, Jeong-Hui Park ${ }^{1,2}$, Rajendra K. Singh ${ }^{1,2,5}$, \\ Jun-Hyeog Jang ${ }^{6}$, Ueon Sang Shin ${ }^{1,2, *}$, Jonathan C. Knowles ${ }^{2,5,7,8}$, Hae-Won Kim ${ }^{1,2,3,4,5, *}$
}

${ }^{1}$ Institute of Tissue Regeneration Engineering (ITREN), Dankook University, Cheonan 31116, South Korea ${ }^{2}$ Department of Nanobiomedical Science \& BK21 NBM Global Research Center for Regenerative Medicine, Dankook University, Cheonan 31116, South Korea

${ }^{3}$ Department of Biomaterials Science, College of Dentistry, Dankook University, Cheonan 31116, South Korea ${ }^{4}$ Cell \& Matter Institute, Dankook University, Cheonan 31116, Republic of Korea

${ }^{5}$ UCL Eastman-Korea Dental Medicine Innovation Centre, Dankook University, Cheonan 31116, South Korea ${ }^{6}$ Department of Biochemistry, College of Medicine, Inha University, Incheon, Republic of Korea ${ }^{7}$ UCL Eastman Dental Institute, University College London, 256 Gray's Inn Road, London WC1X 8LD, UK ${ }^{8}$ The Discoveries Centre for Regenerative and Precision Medicine, UCL Campus, London, UK

\footnotetext{
${ }^{\S}$ These authors contributed equally to this work.

*Corresponding authors:

Shin US (Tel: +82 41550 3691, Fax: +82 41559 7911; E-mail: usshin12@ dankook.ac.kr)

Kim HW (Tel: +82-41-550-3082, Fax: +82-41-550-3085; E-mail: kimhw@dku.edu)
} 


\section{<Graphical abstract>}

(Multifunctional nanohybrid self-assembled from core-shell nanounits)
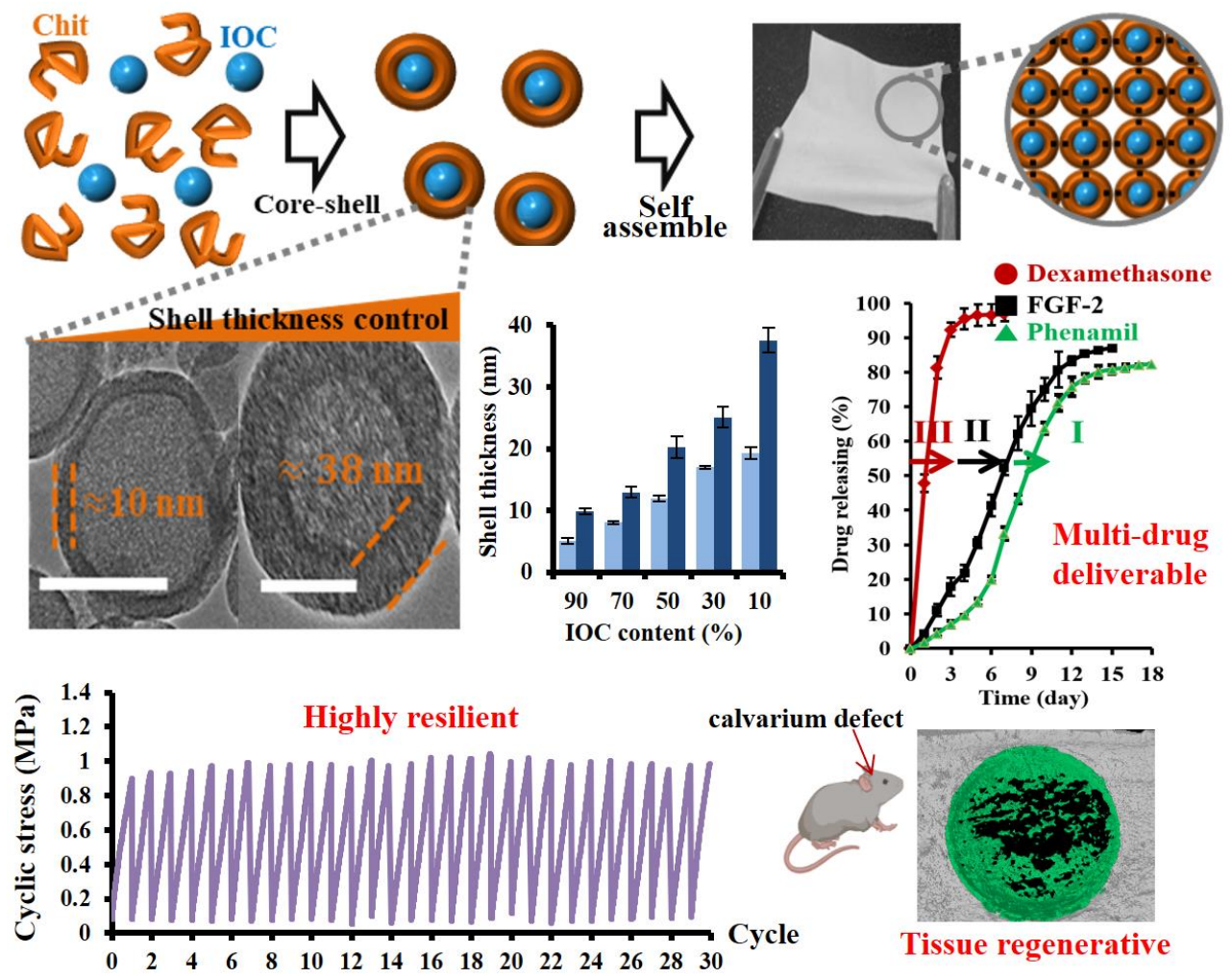


\begin{abstract}
Natural inorganic/organic nanohybrids are a fascinating model in biomaterials design due to their ultramicrostructure and extraordinary properties. Here, we report unique-structured nanohybrids through selfassembly of biomedical inorganic/organic nanounits, composed of bioactive inorganic nanoparticle core (hydroxyapatite, bioactive glass, or mesoporous silica) and chitosan shell - namely Chit@IOC. The inorganic core thin-shelled with chitosan could constitute as high as $90 \%$, strikingly contrasted with the conventional composites. The Chit@IOC nanohybrids were highly resilient under cyclic load and resisted external stress almost an order of magnitude effectively than the conventional composites. The nanohybrids, with the nanoroughened surface topography, could accelerate the cellular responses through stimulated integrin-mediated focal adhesions. The nanohybrids were also able to load multiple therapeutic molecules in the core and shell compartment and then release sequentially, demonstrating controlled delivery systems. The nanohybrids compartmentally-loaded with therapeutic molecules (dexamethasone, fibroblast growth factor 2, and phenamil) were shown to stimulate the anti-inflammatory, pro-angiogenic and osteogenic events of relevant cells. When implanted in the in vivo calvarium defect model with 3D-printed scaffold forms, the therapeutic nanohybrids were proven to accelerate new bone formation. Overall, the nanohybrids self-assembled from Chit@IOC nanounits, with their unique properties (ultrahigh inorganic content, nano-topography, high resilience, multiple-therapeutics delivery, and cellular activation), can be considered as promising 3D tissue regenerative platforms.
\end{abstract}

Keywords: Self-assembly; inorganic-organic nanohybrids; core-shell; ultrahigh inorganic content; resilience; controlled drug delivery; tissue regeneration 


\section{Introduction}

In bioengineering field, natural and biological inorganic/organic hybrids are often a fascinating model to mimic the nano/micro-structure due to their unprecedented physico-chemical and mechanical properties[1-4]. Teeth, bones, mollusk shells, and clay minerals are the common examples of natural hybrids where the organic components act as strong adhesives and biological activators while the inorganic nanocomponents serve as rigid building blocks. In such systems, the inorganic nanocomponents are fully integrated with organic components to form highly ordered nanohybrid structures, which ultimately contributing to the extraordinary properties of the materials (e.g., ultrahigh strength, toughness, elongation, etc.)[5, 6]. Although substantial progress has been made in developing inorganic-organic hybrids that mimic natural materials[7-23], challenges still remain; among others, the inorganic nanocomponents are not homogenously intercalated with the organic part, limiting the content of inorganic phase and the resultant properties[24, 25]. To address this issue, significant effort has been made. For example, the inorganic nanoparticles were modified with functional (phosphate or amine) groups[26-29] or coated with organics using solvent or spray-coating process[30, 31], which however often holds time-consuming steps, expensive equipments, and/or harsh processing environments (e.g., toxic chemicals, high temperature, dramatic change in acidity), limiting the potential utility as biomedical materials.

Herein, we aim to develop biomedical hybrids made of natural organic phase and homogeneously intercalated bioactive inorganic nanoparticles (hydroxyapatite, bioactive glass, or mesoporous silica) that can allow ultrahigh content of an inorganic component, through a simple process under mild aqueous conditions. For this, we introduced chitosan as the organic phase that acts as a bioadhesive to cover (or coat) the individual inorganic nanoparticles uniformly. The inorganic nanoparticle enveloped with chitosan thus features a coreshell structure, and the core-shell hybrid nanounits can further assemble into nanohybrids in the form of either compacted membrane or coating on 3D scaffolds. We examined the physico-chemical and mechanical properties resulting from the unique self-assembled nanostructure in comparison with conventionally mixed composites. We further designed the nanohybrids for loading of multiple therapeutic molecules and then delivery in a controlled (sequential) manner, to enhance the therapeutic potential. We investigated the interactions of the nanohybrids with diverse sets of cells, and evidenced their therapeutic efficacy in the in vivo bone formation model, to address the potential usefulness as $3 \mathrm{D}$ tissue regeneratives.

\section{Results and Discussion}

\section{Core-shell nanounits self-assemble to nanohybrids with ultrahigh inorganic content}

We first synthesized a core-shell structured nanounits made of inorganic nanoparticle (IOC) and thin chitosan shell that were used as the building blocks for the self-assembled nanohybrids. As depicted in Figure 1A, the chitosan molecules dispersed in an aqueous solution at low $\mathrm{pH}$ (acidic) became adhesive with increasing $\mathrm{pH}$ (neutral or basic) through hydrogen bonds, during which they adhered to the surface of IOC (hydroxyapatite (HA), bioactive glass nanoparticle (BGn) or mesoporous silica nanoparticle (MSN) used as model IOC) to 
generate core-shell hybrid nanounits. More specifically, the amine groups $\left(-\mathrm{NH}_{2}\right)$ in chitosan convert to ammonium groups $\left(-\mathrm{NH}_{3}{ }^{+}\right)$under acidic conditions, resulting in a homogenous dispersion, whereas, under neutral or basic conditions, a deprotonation regenerates amine groups, which leads to an assembly of chitosan molecules on the surface of IOC. The obtained chitosan shell / IOC core nanospheres (namely, Chit@IOC) were then structured to nanohybrids through a self-assembly process on a supporting substrate during evaporation, where the shape of nanohybrids could largely depend on the type of substrate, i.e., formed into a bulky membrane on a flat glass or coated on a surface of 3D fibrous / porous scaffold at a controlled amount.

It is worth noting that through this process the inorganic content in the nanohybrids could reach as high as to $90 \mathrm{wt} \%$. As deduced from summarized data (Figure S1, Table S1, Supporting Information), the $90 \%$ of inorganic content is almost maximized one; previous efforts could achieve nanocomposites with much less content of inorganic particles, but only some of the natural systems can contain such a high content of an inorganic phase. This is mainly due to that the organic phase is poorly interspaced between the inorganic nanocomponents, resulting in aggregated inorganic nanoparticles devoid of organic phase. In fact, through a conventional mixing of chitosan molecules with inorganic nanoparticles (namely 'Chti+HA', 'Chit+MSN', or 'Chit+BGn'), only up to 30 50wt\% of inorganic phase could be added, which strikingly contrasted with the nanohybrid 'Chit@HA', ‘Chit@MSN', or ‘Chit@BGn' produced by the novel method approached herein (Table S2 and Figure S2, Supporting Information).

The nanoscale core-shell morphology of the Chit@IOC with different compositions was examined by TEM (Figures 1B \& also Figure S3, Supporting Information). The TEM images revealed well the core-shell structured hybrid nanoparticles where the chitosan enveloped the inorganic nanoparticle quite uniformly. The FE-SEM images of the nanohybrids with representative composition (50\% IOC) showed the inner core inorganic morphology was well preserved after the chitosan coating (Figures 1B). The shell thickness on the inorganic core was controllable by adjusting the ratio of Chit and IOC content during reaction. The shell thickness was measured to range from 5 to $19 \mathrm{~nm}$ for Chit@HA (Figure 1C) and from 10 to $38 \mathrm{~nm}$ for Chit@MSN (Figure S4, Supporting Information), and the thickness of the Chit shell correlated well with the content of Chit. Based on the observations, the $5 \mathrm{~nm}$ and $10 \mathrm{~nm}$ thickness is considered as the thinnest Chit layer to exploit, respectively for HA and MSN, which ultimately amounts to $90 \mathrm{wt} \%$ of the inorganic content used. The actual contents of inorganic nanoparticles, as confirmed by TGA, corresponded well to those as designed (Figure S5, Supporting Information), suggesting the Chit and inorganic nanoparticles reacted almost completely to self-assemble the resultant nanohybrids. Due to the Chit shell formed on the surface of IOC, the surface $\zeta$-potential of the hybrid nanounits shifted from negative toward positive (Figure S6, Supporting Information). Another notable characteristic of the Chit@IOC nanohybrids is the flexibility and shape formability. As revealed in Figure 1D, the nanohybrids prepared in a membrane form were found to be foldable, stretchable, and perforable regardless of the type of IOC, implying the possible applications as surgical implants, i.e., surgical-friendly biomaterials. The nanohybrids could also cover the surface of complex-shaped materials such as nanofibers and porous 3D scaffolds at controlled contents regardless of the type of IOC, suggesting the nanohybrids can be used as the coating layer to alter the surface nanostructure and compositional properties of the underlying 3D implantable biomaterials (Figure 1E). 


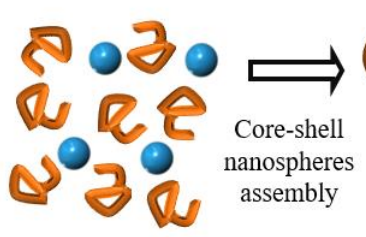

(Acidic condition)

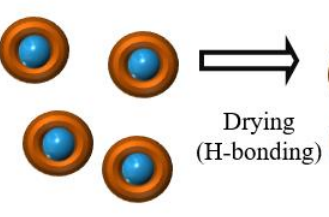

(Neutral condition)

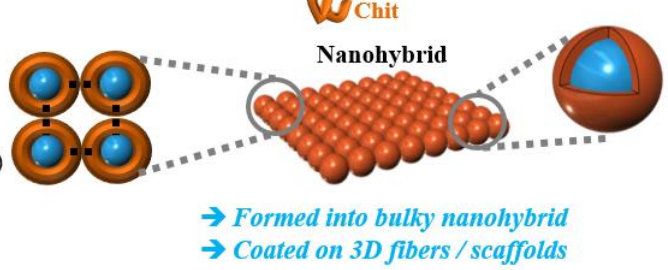

B
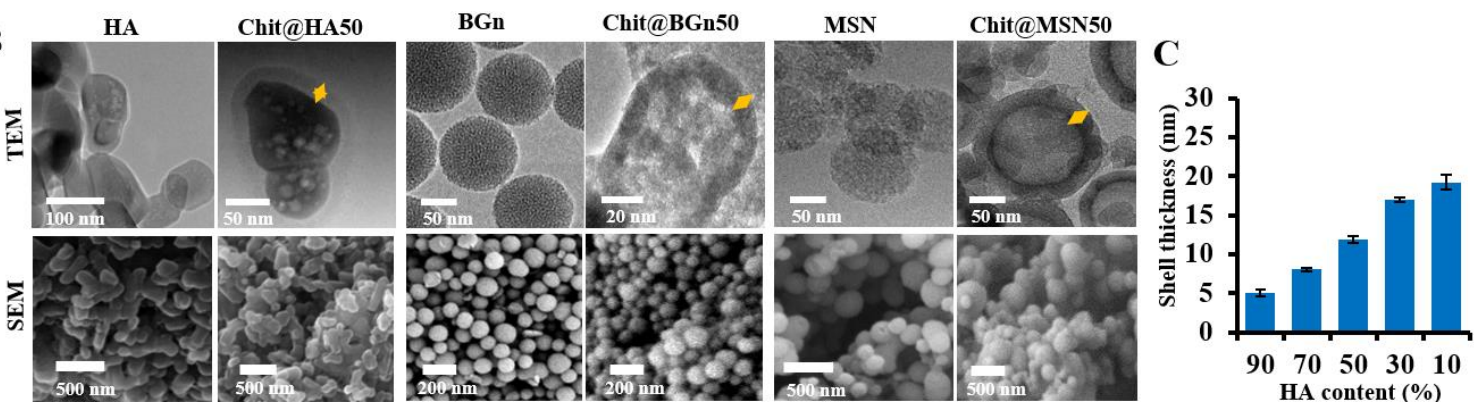

D Nanohybrid bulky membrane

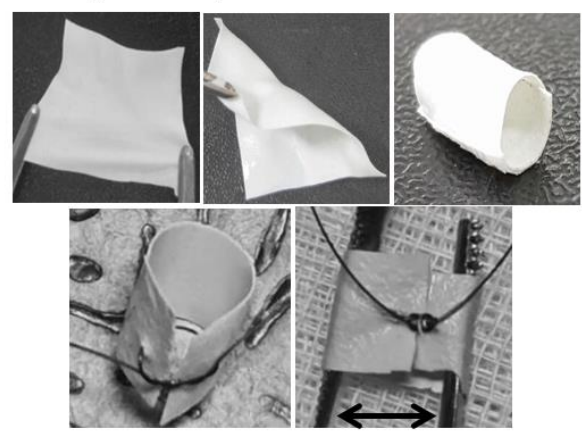

E Nanohybrid coating on $3 D$ structure

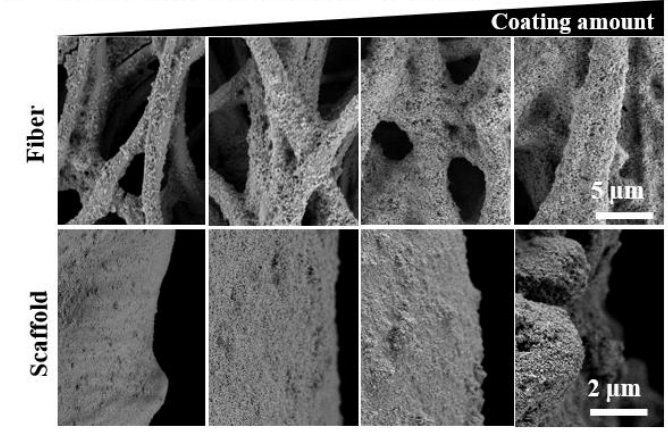

Figure 1. Processing and general characteristics of the self-assembled nanohybrids composed of chitosan and inorganic oxide nanoparticles (IOC): (A) Schematic showing the processing of nanohybrids selfassembled from Chit@IOC core-shell nanounits. (B) TEM and SEM images of the Chit@IOC with different compositions of IOC (hydroxyapatite, bioactive glass, and mesoporous silica). (C) Shell thickness, measured to range from 5 to $19 \mathrm{~nm}$ (Chit@HA shown representatively). (D) Mechanical features of the developed Chit@IOC nanohybrids (Chit@MSN shown representatively); stretchable, foldable, perforable, and suturable, demonstrating the possible applications as implantable biomaterials that need surgical treatments, i.e., surgicalfriendly biomaterials. (E) Potential of the Chit@IOC to form a thin coating layer on complex-shaped materials such as 3D fibers and porous scaffolds at controlled contents to alter the surface nanostructure and compositional properties of the underlying biomaterials (Chit@MSN and Chit@BGn shown representatively for fiber and scaffold coating, respectively).

\section{Nanohybrids exhibit unique physico-chemical and mechanical properties}

The Chit@IOC nanohybrids exhibited highly roughened nanotopography based on FESEM and AFM images (Chit@BGn70 and Chit@HA70 shown representatively in Figure 2A and S7, Supporting Information, respectively), reflecting well the core nanoparticle morphology underneath the ultrathin chitosan shell phase. The nano-roughness of nanohybrid (35.26 nm for Chit@BGn70 and 45.48 nm for Chit@HA70) was 6-8 times higher than that of pure Chit $(6.2 \mathrm{~nm})$. Such a highly roughened nanotopographical feature of the nanohybrids implies the possible effect on biological interactions particularly on the early cell adhesion events[32, 33]. 
Next we analyzed the self-assembled nanohybrids (Chit@BGn analyzed representatively) by XPS. On a wide scan (Figure S8A, Supporting Information), the Chit-related peaks at 286.2 (C 1s), 400.0 (N 1s) and $533.5 \mathrm{eV}$ (O 1s) and the BGn-related peaks at 347.5 (Ca 2p) and $104.5 \mathrm{eV}$ (Si 2p) were detected well for all the nanohybrid compositions. On a narrow scan (Figure S8B, Supporting Information), the carbon (C 1s) in Chit revealed two peaks ( $\mathrm{C}-\mathrm{C}$ and $\mathrm{C}-\mathrm{O}$ ) in the nanohybrid while the $\mathrm{O} 1 \mathrm{~s}$ and $\mathrm{N}$ 1s peaks showed some shifts in binding energy depending on the nanohybrid composition. Also, the peaks of BGn-related elements (Ca $2 p$ and Si 2p) exhibited shifts in binding energy. Of note, such shifts in binding energy (by $0.5 \sim 1.7 \mathrm{eV}$ ) of elements (O 1s, N 1s, Ca 2p, and Si 2p), particularly toward lower values relative to pure Chit (for O 1s and N 1s) and pure BGn (for $\mathrm{Ca} 2 \mathrm{p}$ and $\mathrm{Si} 2 \mathrm{p}$ ), are indicative of newly formed chemical interactions between the electron poor/rich ions of $\mathrm{BGn}\left(\mathrm{Ca}^{2+} / \mathrm{SiO}^{4+} / \mathrm{OH}^{-}\right)$and the highly polar functional groups of the $\mathrm{Chit}(\mathrm{C}=\mathrm{O}, \mathrm{N}-\mathrm{H}$, and $\mathrm{O}-$ H).[34, 35] Unlike the self-assembled Chit@BGn nanohybrids, the conventionally mixed composites (Chit+BGn) did not show such a clear shift, especially in N 1s, Ca 2p and Si 2p binding energy (as compared in Figure 2B). The shifts in binding energy of elements ( $\mathrm{O}, \mathrm{N}, \mathrm{Ca}$ and $\mathrm{P})$ toward lower values relative to pure Chit were similarly noted in Chit@HA nanohybrids (Figure S9, Supporting Information), which was rarely observed in conventional Chit+HA composite (Figure S10, Supporting Information). The XPS results highlight the chemical interactions between Chit and IOC, which contrasted with the conventional composites.

The Chit@IOC nanohybrids exhibited an increased hydrophilicity relative to pure Chit, which was more noticeable as the IOC content increased (Figure S11, Supporting Information). The innate hydrophilic property of the IOC (BGn and HA) as well as the surface nano-roughened topography of the nanohybrids might influence the increased hydrophilicity. The nanohybrids also showed a level of water uptake capacity - a characteristic of the shell, natural polymer Chit. As the IOC content increased, the water-uptake capacity was thus decreased (Figures S12, Supporting Information). The observed properties of the nanohybrids - reduced swelling behavior with increased hydrophilicity - are merited for the applications as stable implantable biomaterials. Next, we measured the hydrolytic degradation (in PBS at $37^{\circ} \mathrm{C}$ ) of the Chit@IOC nanohybrids over 4 weeks. For Chit@BGn, the degradation increased with increasing BGn content, and the weight change for 4 weeks was recorded to be higher $(\sim 10-20 \%$ up to $70 \% \mathrm{BGn})$ than that of pure Chit $(\sim 6 \%)$; for the Chit@BGn90 the degradation was very rapid ( 100\% over 4 weeks) (Figure S13A, Supporting Information). In case of Chit@HA, the degradation was also higher than that of pure Chit, which was even with 90\%HA (Figure S13B, Supporting Information). The hydrolytic degradation property of IOC might reflect the degradation of nanohybrids, i.e., BGn degrades more quickly than HA.

We next investigated the tensile mechanical properties of the Chit@IOC nanohybrids under wet conditions. For the applications as implantable biomaterials, favorable surgical handling with elastic deformation and longterm adaptation to defects under a dynamic force are some of the key requirements. As provided in the representative experimental movies (Videos S1\&2, Supporting Information), the Chit@HA30 nanohybrid resisted the applied tensile force more effectively than the Chit+HA30 conventional composite. Quantitative analyses of the mechanical properties were then carried out by comparing Chit@BGn and Chit+BGn. First, the tensile strengths of the Chit@BGn nanohybrids recorded (0.99 3.20 MPa) were significantly higher than those of Chit+BGn composites (0.18 0.31 MPa), by almost an order of magnitude difference (Figure 2C). 
The tensile strength showed a bimodal behavior peaking at $~ 50 \%$ BGn while the elastic modulus of the nanohybrids gradually increased with increasing BGn content (Figure 2D) - a behavior generally observed in the organic-inorganic nanocomposites in other biological and engineering systems[36]. Of note was the elongation of nanohybrids (Figure 2E); the Chit@BGn nanohybrids were highly elongated (74 403\% elongation) versus the Chit+BGn conventional composites were easily broken to show limited elongation (34 40\%), demonstrating a highly resilient property of the nanohybrids. Such mechanical behaviors observed in Chit@BGn were similarly demonstrated in Chit@HA and Chit@MSN (Figure S14A,B, Supporting Information). The poor mechanical properties of the conventional composites are attributed to the aggregated inorganic particles without a homogenous Chit coverage which potentially act as stress generator and failure origin. The observed excellent mechanical properties highlight that both organic and inorganic components in the self-assembled nanohybrids, with their hierarchical organization at the nanoscale, contribute effectively to the resistance to a failure under a tensile stress. Under dry conditions of the samples, a similar trend in the mechanical properties was also detected (Figure S15, Supporting Information).

Next, we examined the mechanical behaviors of the nanohybrids under dynamic stress conditions. The sample that has shown the highest tensile strength (Chit@BGn50) was chosen and a cyclic tensile load was applied up to 30 cycles $(10 \%, 5 \mathrm{MPa} / \mathrm{min})$. A continuous elastic behavior with a full recovery within a few seconds was observed over the 30 cycles of the load (Figures 2F). A similar cyclic behavior was also observed in other nanohybrids, Chit@HA and Chit@MSN (Figure S14C, Supporting Information). The results demonstrate that the Chit@IOC are highly resilient and flexible to resist against a failure under a cyclic tensile load, which supports their potential applications as implantable biomaterials with surgical handling and the long-term clinical adaptation under dynamic force conditions, which yet needs further confirmation in the in vivo cyclic loading environments. 
A
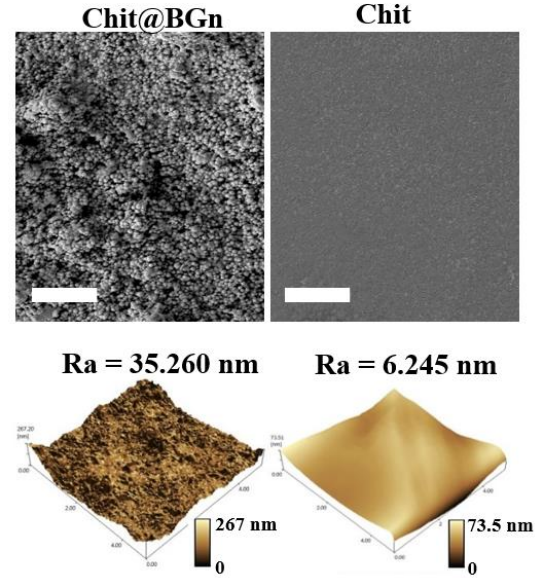

B
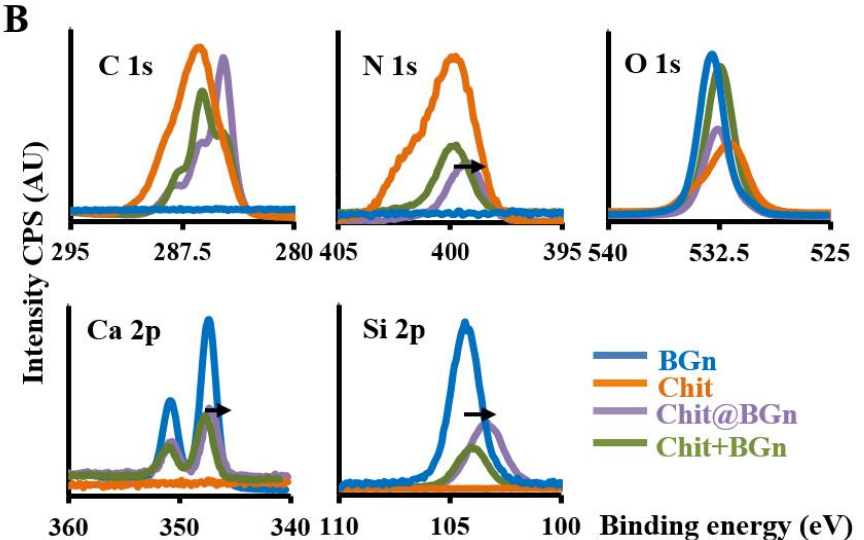

C

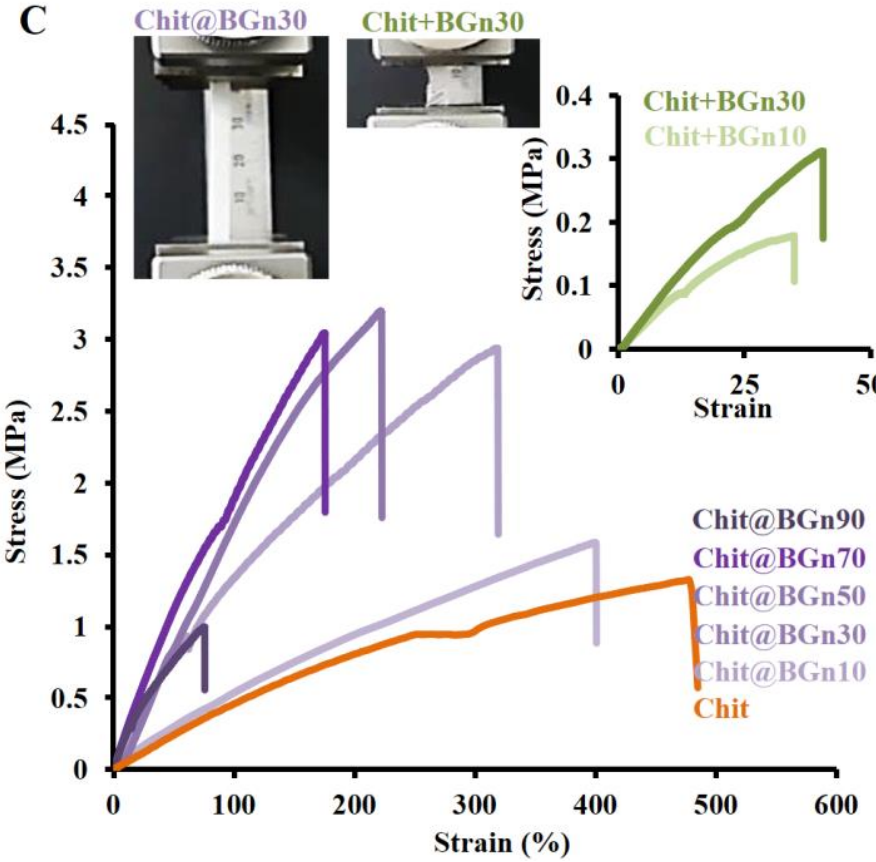

D

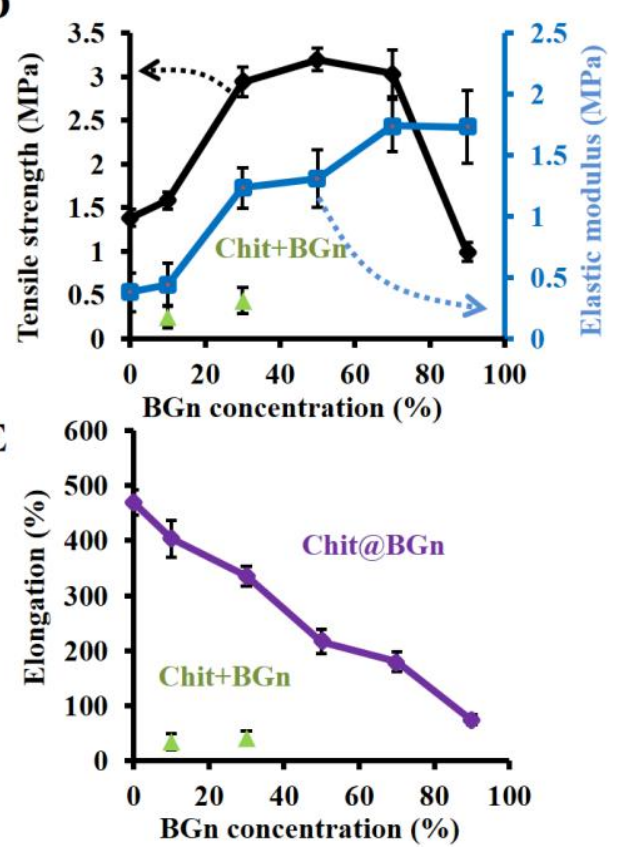

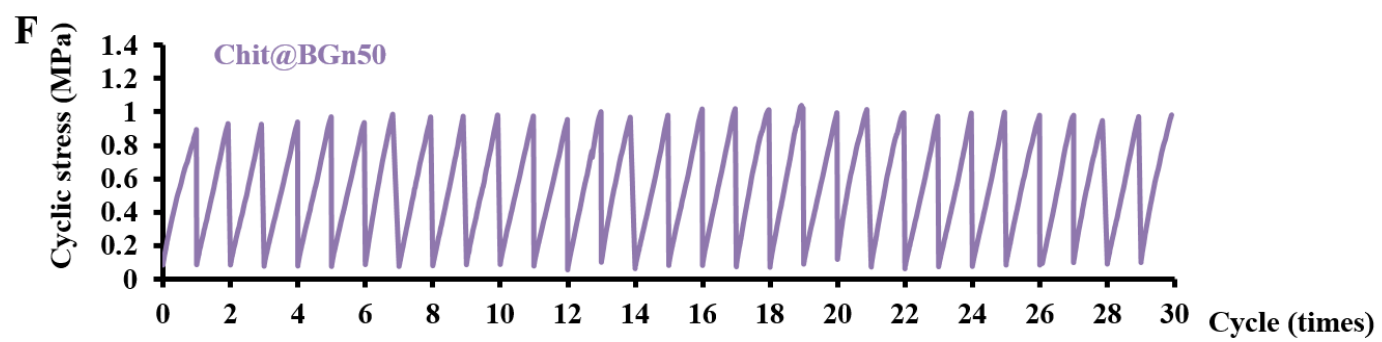

Figure 2. Physico-chemical and mechanical properties of the nanohybrids: Chit@BGn shown representatively. (A) FESEM and AFM surface images of nanohybrid, exhibiting highly roughened nanotopographical surface, with a roughness value of $35.26 \mathrm{~nm}$ for Chit@BGn vs. $6.25 \mathrm{~nm}$ for pure Chit, showing $\sim 6$ times difference. (B) XPS scanned at a narrow scale revealing that the self-assembled nanohybrid Chit@BGn has a clear shift in binding energy (by $0.5 \sim 1.7 \mathrm{eV}$ ) toward lower values relative to pure chitosan (for $\mathrm{O} 1 \mathrm{~s}$ and $\mathrm{N} 1 \mathrm{~s}$ ) and pure $\mathrm{BGn}$ (for $\mathrm{Ca} 2 \mathrm{p}$ and $\mathrm{Si} 2 \mathrm{p}$ ), indicative of newly formed chemical interactions between electron poor/rich ions of BGn and highly polar functional groups of Chit, which contrasted with the conventional composite Chit+BGn. (C) Static tensile mechanical behaviors of the nanohybrids under wet conditions, presented as stress-strain curves. Insets are the typical images of test samples, exhibiting highly elongated nanohybrid vs. catastrophically failed conventional composite. The stress-strain curves of 
conventional composites are also compared (note the remarkable difference in stress and strain scales of the Chit@BGn and Chit+BGn graphs). (D) Elongation of the nanohybrids ranges from $403 \%$ (for $10 \% \mathrm{BGn}$ ) to $74 \%$ (for $90 \% \mathrm{BGn}$ ), significantly higher than the conventional composites (elongation $\sim 34-40 \%$ ) at the corresponding BGn content (as marked in green triangle symbol). (E) Tensile strength and elastic modulus of the Chit@BGn nanohybrids, showing striking differences from those of conventional composites, by an order of magnitude (strengths of conventional composites are marked in green triangle symbol). (F) Dynamic mechanical behavior of the nanohybrid (Chit@BGn50 shown representatively) under cyclic tensile loading conditions (30 cycles, $10 \%$ strain, $5 \mathrm{MPa} / \mathrm{min}$ ); A continuous elastic behavior with a full recovery within a few seconds over the 30 cycles of load demonstrates the excellent flexibility of the nanohybrid to resist against a failure under a cyclic tensile load, implying the possible applications as implantable biomaterials with surgical handling and the long-term clinical adaptation under dynamic force conditions.

\section{Nanohybrids activate adhesion-dependent cellular process and support tissue repair}

To address the biological efficacy of the Chit@IOC nanohybrids as implantable biomaterials, we first investigated the interactions with cells in vitro using Chit@HA. Among other properties, our interest was focused on the nano-roughened topography of the nanohybrids. As schematically shown in Figure 3A, the cells (here mesenchymal stem cells 'MSCs' are considered) would sense the underlying nano-topography and then adopt their subsequent processes such as adhesion, spreading and differentiation. We used Chit@HA as the model nanohybrid in the nano-topography-related cell and tissue experiments; compared to HA, more soluble BGn might complicate the nano-topography-associated effects on cells, particularly at long-term periods. We cultured MSCs on the representative nanohybrid Chit@HA70 in a growth medium for 4 h or 24 $\mathrm{h}$, and analyzed the cell adhesion behaviors. At $4 \mathrm{~h}$, the nanohybrid significantly increased the number of adhered cells and the spreading area when compared to pure Chit (Figure 3B,C). With respect to TCP control, the nanohybrid showed a comparable adherent cell number while slightly limiting the cell spreading. At $24 \mathrm{~h}$, the adhered cells on the nanohybrid did not show much change in the spreading area whereas those on pure Chit spread substantially at a much lower cell adherent number (Figure S16, Supporting Information). When Chit@BGn70 was used, such an initial cell adhesion behavior was also similarly observed (Figure S17, Supporting Information). The results demonstrate that the activated cellular adhesion at early periods is due to the nano-roughened topography of the nanohybrids, and this is a general phenomenon regardless of the type of IOP.

To determine the biological mechanism underlying the activated adhesion events, we performed $q$ PCR array at $2 \mathrm{~h}$ of culture and analyzed the data based on the mechanotrasduction-related genes including integrin sets, focal adhesion proteins, $\alpha$-actin, linkers of nucleoskeleton and cytoskeleton (LINC) complex, and nucleus lamin A/C (Figure 3D). Nanohybrid sample significantly enhanced the gene expression of integrin sets (integrin $\alpha 2, \alpha 3$, and $\beta 1$ ) as well as adaptor proteins (talin and focal adhesion kinase) that link the integrins to actin filaments, when compared to Chit, demonstrating the altered initial adhesion events of MSCs on the nanohybrid were largely mediated through the integrin (specifically, $\alpha 2 \beta 1$ - and $\alpha 3 \beta 1$ )-dependent focal adhesions[37]. On the other hand, the LINC complex (Sun1, Nesprin1, and Nesprin2) and nucleus lamin A/C, which directly transmit intracellular mechanical signals to a nucleus, were maintained at the same mRNA levels regardless of the substrate, implying these mechanotrasduction machineries might not be strongly 
involved in the events or the possibility of stimulation at protein (not gene) levels, which yet warrants further studies [38, 39]. Albeit such a limitation in analysis tools, the $q \mathrm{PCR}$ array result suggests the nanohybrid should affect the initial cell adhesion number and spreading of MSCs through $\alpha 2 \beta 1$ - and $\alpha 3 \beta 1$-mediated focal adhesions, messaging the possibility of accelerating further osteogenic cascades[40]. As to the reasons for the stimulated adhesion signaling, we consider the significantly altered nano-roughened surface topology (8 times higher nano-roughness) of the nanohybrid ( $v s$. Chit) would contribute primarily while the increased wettability $\left(\sim 50^{\circ}\right.$ vs. $\left.\sim 80^{\circ}\right)$ might also be attributed to. In particular, the nano-roughness level $(45.48 \mathrm{~nm})$ of nanohybrid recorded by AFM is considered to match the size of integrin clusters effective for mechanosensing of the extracellular ligands, i.e., the tens of nanometers of a topographical feature is more effective than the other nanoscale ranges (a few or hundreds of nanometers) for the outside-in mechanosensitive signaling through integrins $[32,38,41]$. The proliferation capacity of the adhered cells during 7 days of culture was also higher in the nanohybrid than in the pure Chit (Figure S18, Supporting Information). Based on these results, the nanohybrid is considered to provide appropriate matrix conditions for the adhesion and growth of stem cells.

Next, we assessed the osteogenic differentiation behavior of MSCs to find the applications of nanohybrids as bone regenerative materials. After 14 days of culture in an osteo-induction medium condition, the bone-related gene expression level (based on ALP) was assessed by $q$ PCR. ALP is essential for providing high concentrations of phosphate at the site of bone mineral deposition, thus considered as an early marker of osteogenesis. A significantly higher ALP expression level ( 2.5-fold) was detected when grown on the nanohybrid than on the pure Chit (Figure 3E). The ALP activity measured at days 7 and 14 further confirmed the time-dependent on-going increase with significantly higher level on the nanohybrid (Figure 3F). The in vitro results demonstrated that the nanohybrid provided excellent extracellular matrix conditions for MSCs in term of initial cell adhesion, proliferation, and osteogenic stimulation which supports the promising applications for bone repair and regeneration.

Following the in vitro study, an in vivo experiment was carried out to examine the interactions with tissues under orthotropic conditions (in a rat calvarium defect model). Two nanohybrid compositions (Chit@HA50 and Chit@HA70 nanohybrid were used whereas Chit@HA90 was ruled out due to its brittle (fragile) mechanical property. made in Samples were made in a thin membrane form and then administered to a rat calvarium defect to guide bone regeneration, and an 'early' neo-bone formation for 5 weeks was examined by $\mu \mathrm{CT}$ and histological staining (as illustrated in Figure 3G). All animals showed favorable osteogenic healing responses without severe inflammatory signs and fibrous tissue ingrowth, suggesting a good tissue compatibility and the role as a barrier against a fibrous tissue invasion, such as a guided bone regeneration (GBR) membrane[42-44]. The $\mu$ CT 3D images showed a neo-bone formation ('NB') within the defect region. The neo-bone formation, albeit not being complete for all groups due to the short healing time (5 weeks), was significantly higher in the nanohybrids than in the pure Chit, in terms of both bone volume and surface area (Figure H,I). The histological observation of the H\&E stained samples revealed the newly formed bone and osteoids in the healing areas (Figure S19, Supporting Information). The neo-bone tissue was observed to grow from the old bone ('OB') margins toward the central defect area. Of note, the neo-bone tissue in the Chit@HA70 group revealed highly populated osteoblasts $(*)$, osteoclasts (\#), and osteocytes (arrowheads), as 
well as trabecular (T) structure, indicative of a typical regenerative process of bone matrix deposition and calcification with simultaneous bone remodeling process[45, 46]. The number of osteoblasts in the lacunae was counted to be significantly higher in the nanohybrids (Figure 3J). Furthermore, the essential bone matrix molecules secreted from the bone forming cells, such as bone sialoprotein (BSP) and ALP, were stained with higher intensities in the nanohybrids (Figure 3K). Taking the in vivo results together, the nanohybrids were proven to be highly effective in accelerating early neo-bone regeneration process, suggesting the potential applications as implantable biomaterials for bone repair and regeneration. While here the in vivo performance of the nanohybrids was investigated in their native form, the bone regenerative capacity will be further accelerated when loaded with candidate therapeutic molecules [47, 48], as demonstrated in the following section. 
A
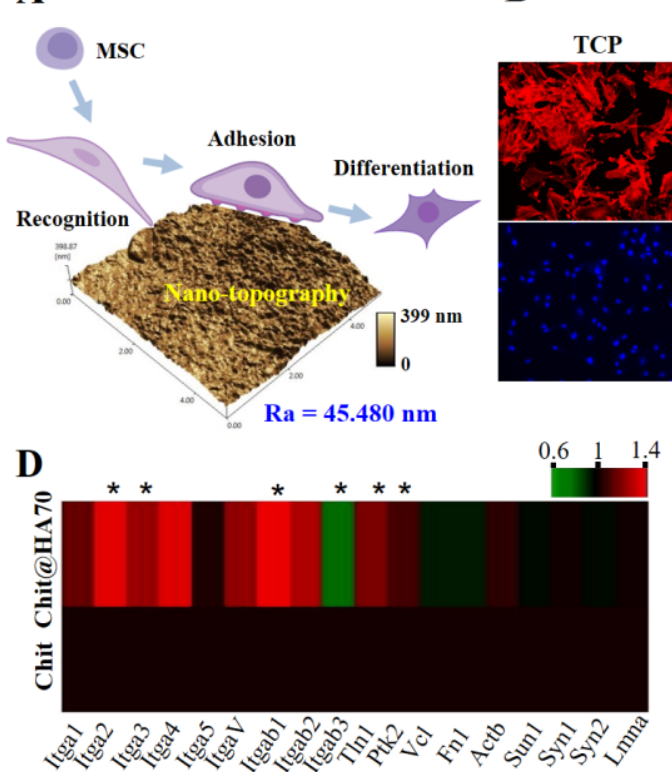

B
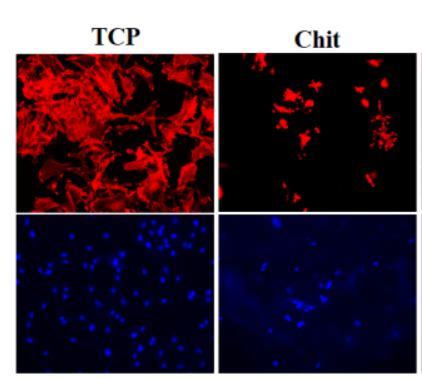

$\mathbf{E}$

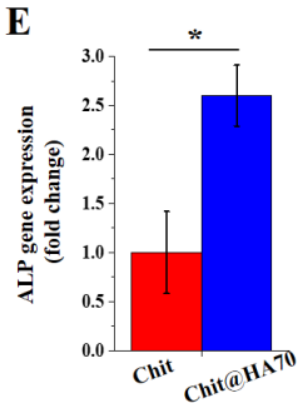

C
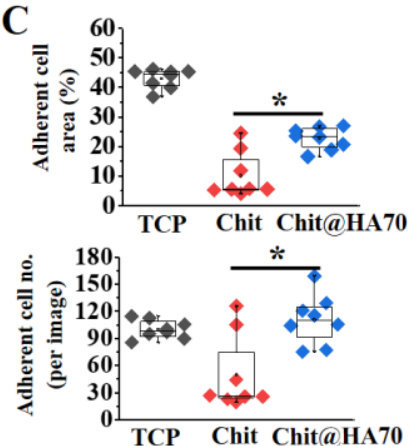

$\mathbf{F}$

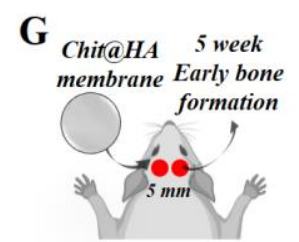

$\mathbf{H}$

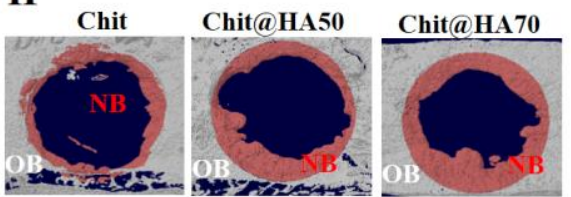

I
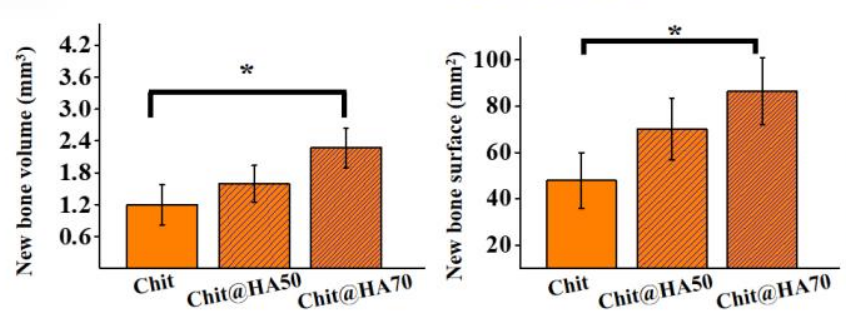

$\mathbf{J}$
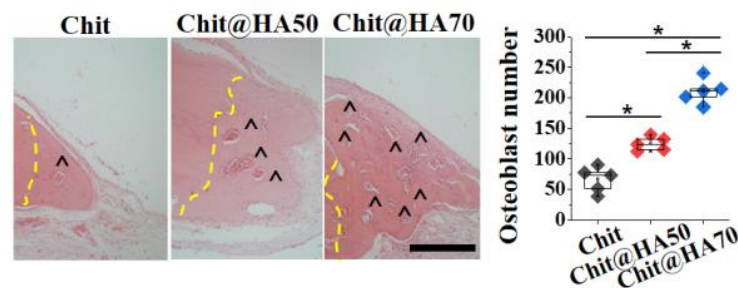

$\mathbf{K}$
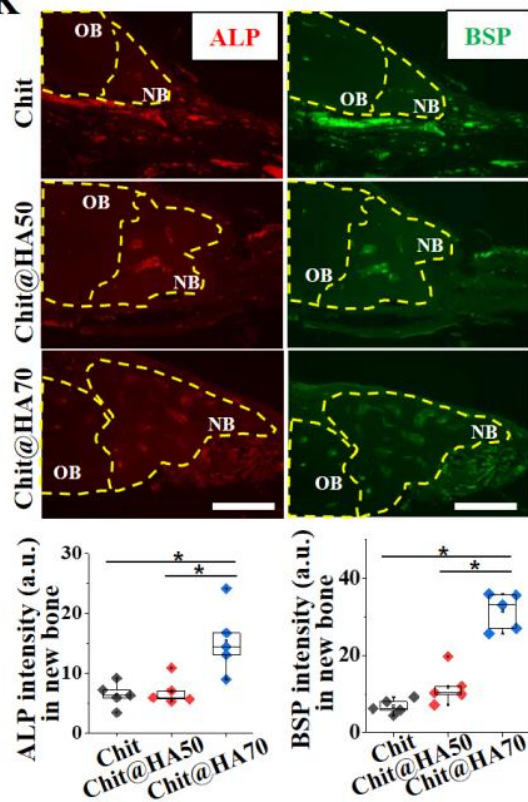

Figure 3. Stem cell activation and early bone formation of the nanohybrid: (A) Schematic showing the MSC activation by the nano-topography surface of nanohybrid. (B) Early (4 h) cell adhesion images with the actin (red) and nucleus (blue) stained, and (C) their quantification in terms of cell adhesion area and adherent cell number. A significant increase in the initial cell adhesion area and number of adhered cells on the Chit@HA70 compared to those on the pure Chit $(\mathrm{n}=8, * \mathrm{P}<0.05)$. (D) $q \mathrm{PCR}$ array revealing adhesion- and mechanotransduction-related gene expression. Chit@HA70 upregulates gene expression of integrin sets (integrin $\alpha 1, \alpha 2, \beta 3$ ) as well as focal adhesion proteins (talin, and protein tyrosine kinase 2) compared to Chit, possibly leading to an increase in initial cell adhesion $(\mathrm{n}=3, * \mathrm{P}<0.05)$. ( $\mathrm{E}$ and $\mathrm{F})$ In vitro osteogenesis at 14 days as measured by alkaline phosphatase (ALP) gene expression $(n=3, * * P<0.01)$ and enzymatic quantity $(\mathrm{n}=3, * \mathrm{P}<0.05)$. (G) Illustrating showing an in vivo experimental design in a rat calvarium defect. Chit@ HA50 and Chit@HA70 were tested as representative nanohybrid groups. (H) $\mu$ CT images of tissue samples implanted for 5 weeks in a rat calvarium tissue. Representative 3D images of new bone, as highlighted in pink (NB = new bone, $\mathrm{OB}=$ old bone). (I) The $\mu \mathrm{CT}$-based quantitative analyses of the newly formed bone, in terms of bone 
volume and surface area (significantly higher level noted in Chit@BGn70 vs. Chit, *p<0.05, n=5). (J) H\&E stained images of tissue samples. Black arrows indicate the margin of the defect area. Enlarged images from the yellow rectangle are shown on the right panel. The border between the old and new bone is indicated by the green dotted line. ( $\mathbf{J})$ Osteoblasts in lacunae of new bone area are highlighted. Osteoblast number is significantly higher in Chit@BGn vs. Chit $(\mathrm{n}=5, * \mathrm{P}<0.05$, ** $\mathrm{P}<0.01)$. (K) ALP (in red) and BSP (in green) expression in tissue samples analyzed by immunohistochemical staining. The yellow line indicates the margin of the hard tissue $(\mathrm{n}=5, * \mathrm{P}<0.05, * * \mathrm{P}<0.01)$. New bone $(\mathrm{NB})$, old bone $(\mathrm{OB})$, osteoblasts $(*)$, osteoclasts (arrowheads). Scale bar is $200 \mu \mathrm{m}$ if not indicated.

\section{Nanohybrids designed for therapeutic-molecules delivering 3D bone regeneratives}

When the nanohybrids can load and deliver therapeutic molecules, the tissue regenerative capacity can be significantly enhanced[47, 48]. Here, we designed the nanohybrids for this purpose, particularly for the delivery of multiple therapeutics and this was based on the unique core-shell structure (and an additional coating process when needed). The loading schemes of two and three different drugs (drug I, II, and III) onto the core-shell structured nanospheres with an additional coating layer (here we used gelatin 'Gel') are schematically shown (Figure 4A). While two different molecules are partitioned in the Gel and Chit@IOC, one more molecule can also be loaded in the mesoporous type of IOC ('MSN or BGn'). In a proof-of-concept experiment, a hydrophobic drug (drug II, paclitaxel denoted 'PTX') was incorporated into the Chit layer, and a hydrophilic drug (drug III, doxorubicin hydrochloride denoted 'DOX') was deposited in the Gel layer by glutaraldehyde. The additional coating of Gel was shown to preserve well the initial morphology of the Chit@IOC (Figure S20, Supporting Information). The release of the loaded drugs was then measured and plotted when referenced to calibration curves (Figure S21, Supporting Information). The dual-drug release profile from Chit@HA50-Gel was then examined. The drug III ('DOX') loaded in the additional Gel layer started to release from day 1 , then showed a linear release rate for up to 7 days, followed by a slow-down in the release rate (Figure 4B). On the other hand, the drug II ('PTX') placed in the internal Chit layer began to release after $\sim 5$ days, which then profiled a gradual release to saturate at $\sim 15$ days. The results clearly demonstrated a time-dependent sequential release of two different drugs from the well-designed nanohybrid delivery system.

Next, we prepared a triple-therapeutics delivery system by loading drug I (Phenamil; 'Phe') within the mesoporous BGn core prior to the preparation of Chit@BGn50-Gel nanohybrid. In this case, FGF2-GFP ('FGF2', drug II) and dexamethasone (denoted 'Dex', drug III) were used as the other two model therapeutic molecules incorporated in the Chit and Gel, respectively. As planned, a time-sequential release of all three molecules was detected; initially, molecule III ('Dex') release from the outermost layer (Gel), then, molecule II ('FGF2') release from the internal Chit layer beginning 3 days later, and lastly, molecule I ('Phe') release from the mesopores of BGn up to 18 days (Figure 4C). Of note, the release behaviors of all three therapeutics were quite gradual without an initial burst and sustainable for days to weeks, suggesting the possible long-term therapeutic efficacy of the molecules released consistently. Furthermore, the release rate of Phe placed in the innermost mesoporous core was controlled to depend on the Chit shell thickness (enabled in Chit@MSN 
composition) where a non-degradable mesoporous core (MSN) was used; the released amount of Phe for 36 days was $290 \%, \sim 66 \%$, and $\sim 27 \%$, respectively for Chit@MSN70-Gel, Chit@MSN50-Gel, and Chit@MSN30-Gel (Figure 4D). The results suggest that the Chit shell can play effectively as a diffusion barrier of the therapeutic molecules placed inside the core, and also that the nanohybrids can be used for controlled therapeutics-delivery systems.

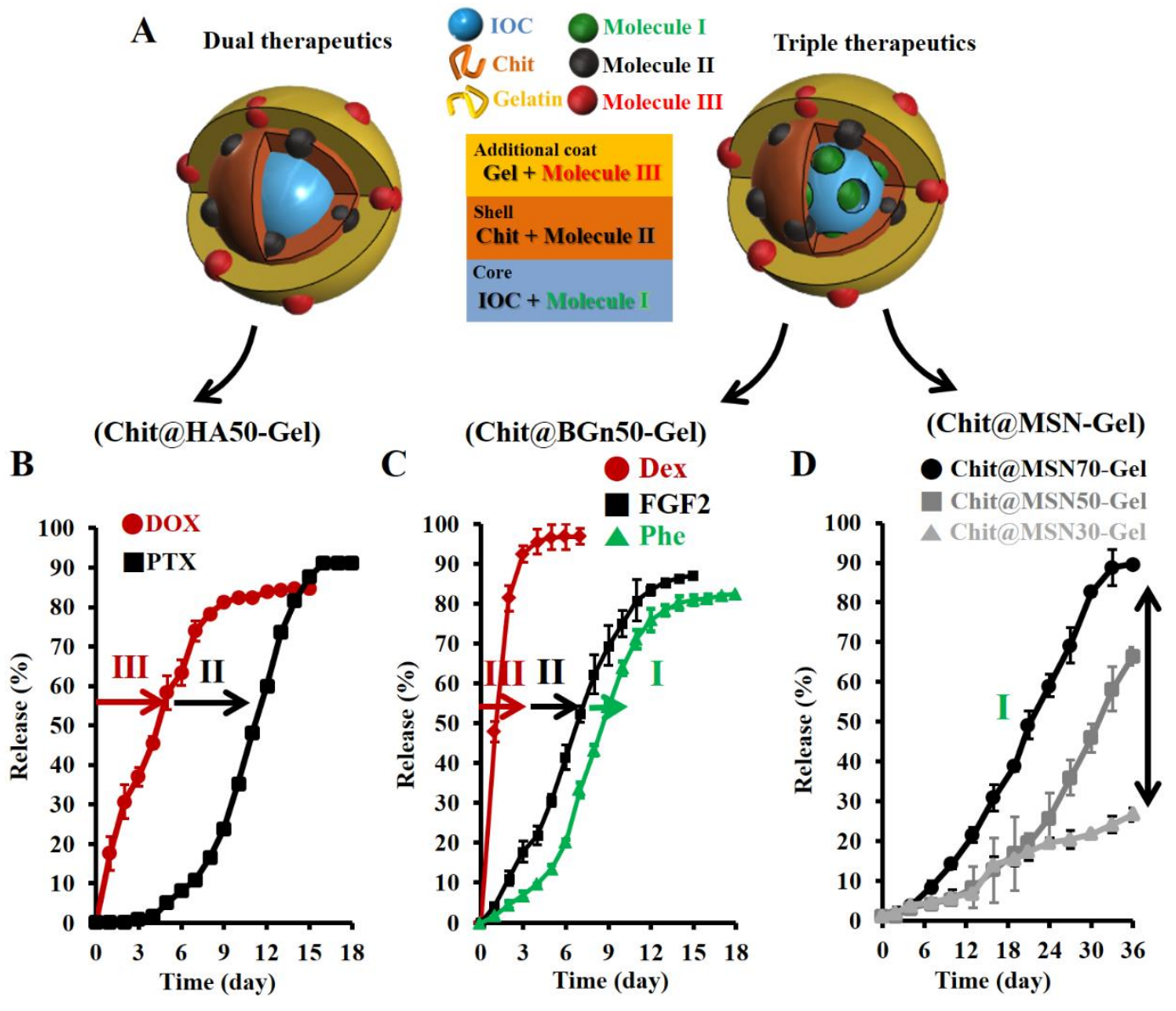

Figure 4. Controlled release of multiple therapeutic molecules from the nanohybrids: (A) Schematic showing the multiple-therapeutics delivery systems. The loadings of two and three different molecules (molecule I, II, and III) onto the core-shell structured nanospheres with an additional coating layer (here we used gelatin 'Gel') are schematically shown. While two different molecules are partitioned in the Gel (III) and Chit@IOC shell (II), one more molecule can also be loaded in the Chit@IOC core using mesoporous type of IOC ('MSN or BGn', I). (B) Sequential dual-therapeutics release profile from the Chit@HA50-Gel; doxorubicin 'DOX' (drug III) in the outermost Gel layer is released first, while paclitaxel 'PTX' (drug II) is trapped inside the Chit shell on the HA core and is released after 5 days. (C) Triple sequential release from the Chit@BGn50-Gel; dexamethasone 'Dex' (drug III) releases first from the Gel layer, then fibroblast growth factor 2 'FGF2' (drug II) releases from the Chit shell, and lastly phenamil 'Phe' (drug I) from the mesopores of the BGn core. (D) Control over the release rate of Phe (drug I) placed in the innermost non-degradable mesoporous core, showing a dependence on the Chit shell thickness (Chit@MSN composition).

Next, we examined the in vitro and in vivo biological efficacy of the nanohybrids as multiple-therapeutics delivery systems. As a representative experimental group, Chit@BGn70 that loads three different therapeutic molecules (Dex, FGF2 and Phe) was tested. As depicted in Figure 5A, the time-dependent sequential release of the three molecules are considered to effectively modulate the biological events (anti-inflammation, pro- 
angiogenesis, and osteo-stimulation) that are required in different time frames of the bone regeneration process. First, the biological function of Dex, which is considered to release relatively quickly, was investigated in terms of anti-inflammatory effect on macrophages cell line (RAW264.7). The macrophages cultured for $4 \mathrm{~h}$ with the extract from the nanohybrid were shown to release less amount of nitric oxide (NO), a proinflammatory marker, and to express significantly lowered pro-inflammatory gene (IL-1 $\beta$ ), demonstrating the biological efficacy of Dex that was released initially from the nanohybrid in subsidizing the inflammatory responses (Figure 5B)[49-51]. One thing to note is the significantly reduced anti-inflammatory signs of cells in the drug-free Chit@BGn70 with respect to those in Chit, which might result from the ions released from the BGn-containing nanohybrid (as deduced from the silicate and Ca ionic release data in Figure S22, Supporting Information), underscoring the Chit@BGn nanohybrids possess additional therapeutic merit related with the ionic release as these ions have previously been shown to be pro-angiogenic and osteo-promotive[49, 52].

Next, we analyzed the angiogenic role of FGF2 (drug II) that would release secondly from the nanohybrid using human endothelial cell line (HUVECs). The HUVECs treated with nanohybrid extract were cultured on a Matrigel-coated culture dish to enable tubule formation. At $12 \mathrm{~h}$, the nanohybrid-treated cells exhibited significantly enhanced tubule formation in terms of circle and node number of tubules, demonstrating the biological role of FGF2 in stimulating endothelial cells (Figure 5C)[52-54]. Lastly, the effect of drug Phe, which would release most slowly, on the osteogenic differentiation was examined using MSCs as the Phe is known as a BMP signaling activator[55, 56]. The MSCs were cultured with the nanohybrid extract in an osteogenic medium and the osteogenic maturation was assessed. When cultured with the Phe-loaded nanohybrid sample for 4 days, the MSCs exhibited significantly higher ALP activity than those cultured with Phe-unloaded one (Figure 5D). Moreover, the expression of late osteogenic marker osteocalcin (OCN) was highly up-regulated by the Phe-releasing nanohybrid (Figure 5E). Taken together, the Chit@BGn nanohybrid loaded with triple molecules (Dex, FGF2, and Phe) demonstrate anti-inflammatory effect on macrophages initially $(\sim 4 \mathrm{~h})$, then angiogenic-activation of endothelial cells $(\sim 12 \mathrm{~h})$, and lastly osteogenic-stimulation of MSCs at prolonged period ( $\sim 7$ days), highlighting the sequential effects of different therapeutic molecules; importantly, this is relevant to the case for bone regeneration where the series of anti-inflammation of macrophages, pro-angiogenesis of endothelial cells, and osteogenesis of resident MSCs would need collectively and/or sequentially.

Following the above in vitro studies, we carried out an in vivo experiment to prove the triple-therapeutics delivery potential in bone regenerative environment. For this, two different compositions (Chit@BGn70 and Chit@BGn70+Tri) were decorated (coated) onto 3D-printed PCL scaffolds, which were implanted in a rat calvarium defect for up to 12 weeks (as illustrated in Figure 5F). The $\mu \mathrm{CT}$ 3D images taken at 12 weeks revealed a significant increase of neo-bone formation (green colored) within the defect region in the Chit@BGn70+Tri group than in the drug-free Chit@BGn70 group (Figure 5G). When quantitated, the neobone formation was significantly higher in Chit@BGn70+Tri than in Chit@BGn70 in terms of both bone volume and surface area, particularly at 12 weeks (Figure 5H). Taken from the in vitro and vivo results, the triple-therapeutics-loaded nanohybrid was proven to accelerate the neo-bone regeneration process, and this might be the collective events of anti-inflammation, pro-angiogenesis, and osteo-stimulation by the released 
therapeutic molecules, taken from the in vitro studies albeit not analyzed in the in vivo samples. Furthermore, the findings suggest that the nanohybrid-based 3D scaffolds can be potentially useful as multiple drug delivery implantable biomaterials for bone repair and regeneration. This concept may also be applicable to a variety of tissues (e.g., skin, muscle, and nerve), not limited to bone, when formulated with proper core compositions and therapeutic molecules for the target tissues where the controlled and sequential delivery of multiple therapeutics would potentially need to accelerate the wound healing and tissue repair process, and this remains further exploration of study. 


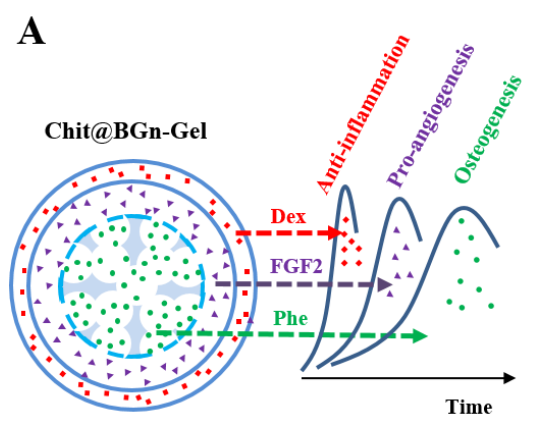

B Anti-inflammation on RAW264.7 (at 4 h)

C

Pro-angiogenesis on HUVEC (at 12 h)
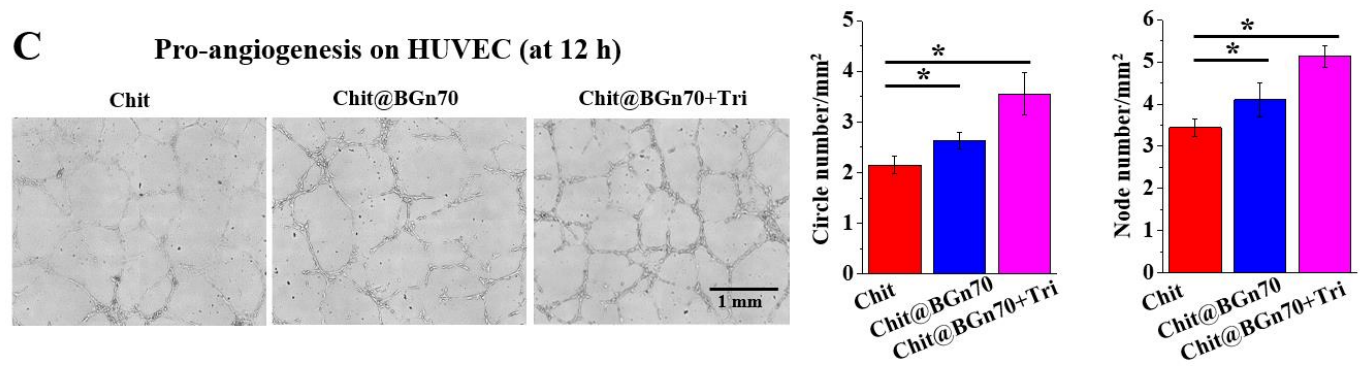

D

Osteogenesis on MSC (at 7 d)
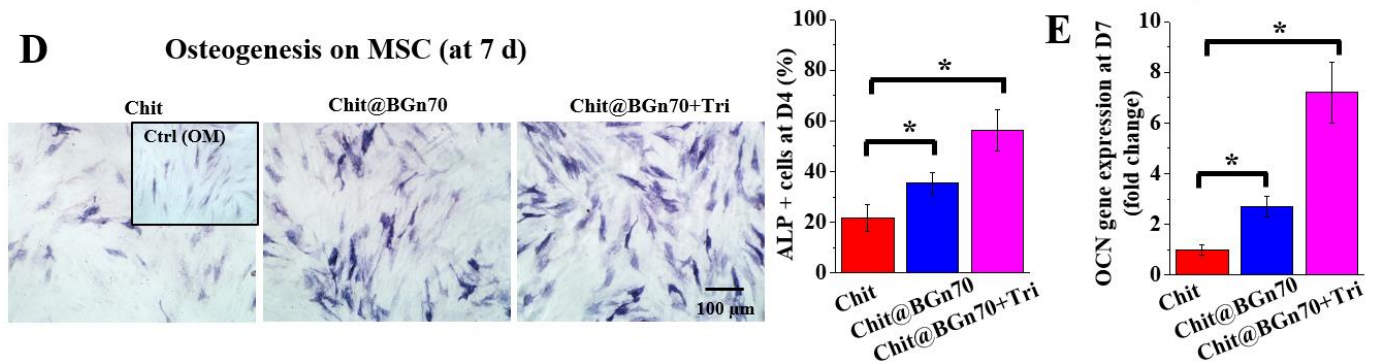

$\mathbf{F}$

F Bone regeneration in rat $\mathbf{G}$ calvarium defect
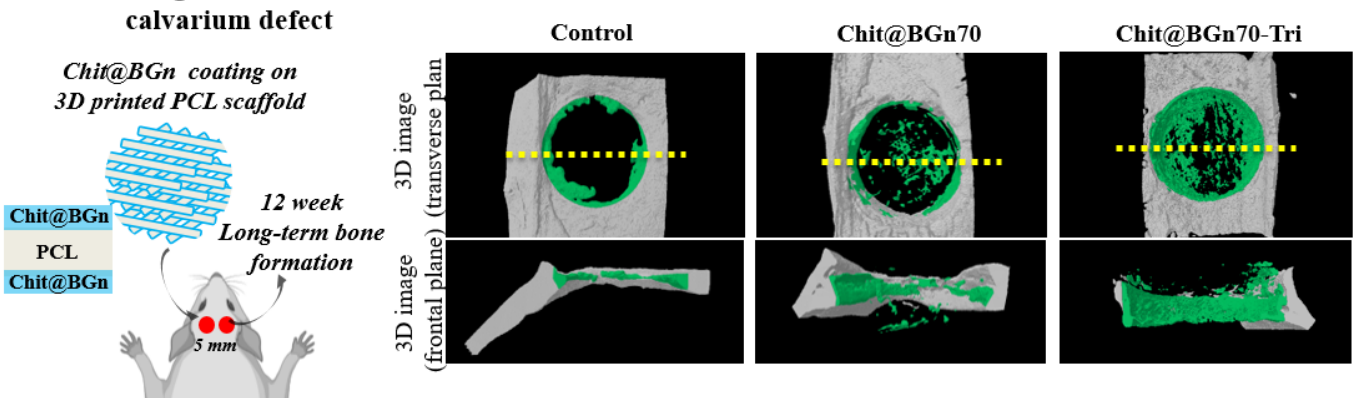

$\mathbf{H}$
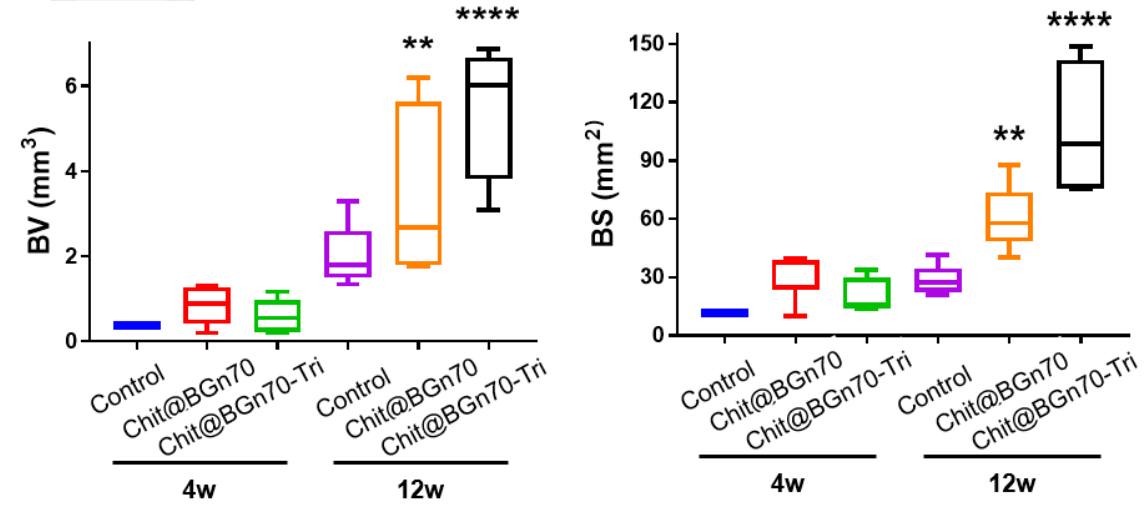

Figure 5. Therapeutic efficacy of the nanohybrid scaffolds loaded with triple-molecules (Chit@ BGn70+Tri) for bone regeneration. (A) Schematic illustrating the time-dependent sequential release of triple-molecules dexamethasone (Dex), FGF2, and phenamil (Phe) for anti-inflammation, pro-angiogenesis 
and osteogenesis, respectively. (B) Anti-inflammatory effect of Chit@BGn70+Tri evidenced in macrophages cell line (RAW264.7) which was enabled by the rapidly releasing drug Dex from the outermost layer. LPSinflamed RAW264.7 cells treated with Chit@BGn70+Tri (extract for $4 \mathrm{~h}$ ) expressed significantly downregulated pro-inflammatory gene (IL-1 $\beta)$ and released decreased level of NO than Chit@ BGn70 $(n=4, \mathrm{P}<0.05)$. (C) Pro-angiogenesis effect of Chit@BGn+Tri on human endothelial cell line (HUVECs) by the released FGF from chitosan layer. Extract from Chit@BGn70+Tri for $12 \mathrm{~h}$ was treated to HUVECs upon Matrigel-coated substrate. Tubule formation (circle and node number) was significantly enhanced in Chit@BGn70 (due to angiogenic silicate ions) and more accelerated in Chit@BGn70+Tri due to FGF release $(n=4, P<0.05)$. $(D, E)$ Osteogenic differentiation of MSCs stimulated by Chit@BGn70+Tri. Release of osteogenic drug Phe from Chit@BGn+Tri was effective in increasing alkaline phosphatase activity at day 4 (D) and up-regulating OCN gene expression at day $7(\mathrm{E})(\mathrm{n}=4, \mathrm{P}<0.05)$. (F) Illustration of in vivo design in a rat calvarial defect model where the nanohybrid coated 3D-printed PCL scaffolds were administered for up to 12 weeks. (G) Neo-bone formation (green colored) examined by $\mu \mathrm{CT}$ within the defect region, representatively shown at 12 weeks. (H) Quantification of $\mu \mathrm{CT}$ constructed images in terms of bone volume and surface area, showing significantly higher levels in Chit@BGn70+Tri scaffold than in drug-free Chit@BGn70 scaffold ( $\mathrm{n}=5$, ** $\mathrm{P}<0.01$, $* * *<0.001)$.

\section{Conclusion}

Here we reported Chit@IOC nanohybrids self-assembled from the core-shell nanounits by a simple methodology under ambient aqueous conditions. The bioactive inorganic content, easily tunable via chitosan shell thickness (a few to tens of $\mathrm{nm}$ ), was maximized to $90 \mathrm{wt} \%$, which strikingly contrasted with the conventional composites that had limited inorganic contents $(30-50 \mathrm{wt} \%)$. The self-assembled nanohybrids were amenable to shape various forms and to decorate the surface of 3D structures to serve the surgical-friendly implantable biomaterials. The nanohybrids exhibited highly elastic and resilient mechanical properties under static and dynamic stress conditions. Furthermore, the nanohybrids significantly accelerated the adhesion of MSCs through nano-topography-induced stimulation of integrin-mediated focal adhesions, and promoted differentiation into an osteoblast lineage. When applied to a calvarium defect in a thin membrane form, the nanohybrids could support tissue repair with enhanced early new bone growth. The nanohybrids were further designed to load multiple therapeutics within the core and shell compartment, which allowed a time-dependent sequential release. The delivery of three different therapeutic molecules (dexamethasone, FGF2, and phenamil) through the nanohybrids led to a series of sequential biological effects in vitro (anti-inflammation, proangiogenesis, and osteo-stimulation), which were collectively proven to coordinate the enhanced in vivo bone regeneration over 12 weeks. Taken all, the novel self-assembled nanohybrids, demonstrating multifunctional unique properties including ultrahigh inorganic content, shape-formability, high resilience, nano-roughened surface, multiple therapeutics delivery, and bone regenerative potential, are considered as promising implantable 3D biomaterial platforms for the regeneration and repair of tissues including bone. 


\section{Experimental Section}

\section{Materials}

Hydroxyapatite (HA, > 90 purity, $113 \mathrm{~nm}$ average size) and chitosan (Chit, molecular weight 190,000-310,000 Da, degree of deacetylation: 75-85\%) were purchased from Sigma-Aldrich (St. Louis, MO, USA). Mesoporous silica nanoparticles (MSN, $\sim 100 \mathrm{~nm}$ size) and bioactive glass nanoparticles (BGn, $\sim 50 \mathrm{~nm}$ size) were prepared according to our previous works[57, 58]. All additional chemicals, including glacial acetic acid, aqueous ammonia, organic solvents, were purchased from Sigma-Aldrich, and used as an analytical grade, unless otherwise specified.

\section{Preparation of nanohybrids Chit@IOC}

Chit@IOC nanohybrid particle consists of individual inorganic core nanoparticle (HA, MSN or BGn) and thin Chit shell. The nanohybrid particle was prepared at different weight ratios of Chit/OC as follows. First, Chit (360, 280, 200, 120, and $40 \mathrm{mg}$ ) was dissolved in $50 \mathrm{~mL}$ of $1 \mathrm{M}$ acetic acid solution, then, 40, 120, 200, 280, and $360 \mathrm{mg}$ of the inorganic component was added to each Chit, and the mixture was homogenized using a homogenizer (NanoDeBee 45-3, Bee International) to prepare acidic Chit@IOC solutions. The acidic Chit@IOC solutions were then slowly neutralized with the addition of $1 \mathrm{M}$ ammonia solution which was dialyzed (molecular weight cut-off $12 \mathrm{k}-14 \mathrm{k}$ Da, Spectrum Laboratories, Savannah, USA) against distilled water for 2 days to remove small molecules, including inorganic side-products. The obtained aqueous Chit@IOC solutions were transferred to Teflon molds, and the solvent was allowed to evaporate under ambient conditions, yielding strong, white, elastic nanohybrids in a thin membrane form (Chit@BGn10, Chit@BGn30, Chit@BGn50,Chit@BGn70, and Chit@BGn90). The shape was also tuned as desired simply by changing the shape of the Teflon molds, such as star-, flower-, and circle-shaped. To remove any remnant impurities, the nanohybrids were soaked in distilled deionized water while shaking overnight and then washed thoroughly.

\section{Coating of nanohybrids on complex-shaped biomaterials}

For the coating of Chit@IOC, nanofiber and foam scaffold were prepared. First, the nanofiber matrix made of poly(caprolactone) (PCL; MW $=80000$, Sigma-Aldrich) was prepared by electrospinning of $12 \% \mathrm{PCL}$ solution dissolved in 1,2-dichloroethane/methanol[59]. A syringe equipped with a 21-gauge needle, a metal collector, and a high-voltage power supply was set $(15 \mathrm{kV}, 10 \mathrm{~cm}$ distance, $0.5 \mathrm{~mL} / \mathrm{h}$ of injection speed). For the preparation foam scaffolds, the salt-leaching method was used. Polyurethane-PCL mixture (1:1) was dissolved at $20 \%(\mathrm{wt} / \mathrm{v})$ in chloroform, which was added to a cylindrical Teflon mold packed with $\mathrm{NaCl}$ particles (size: $200 \sim 500 \mu \mathrm{m}$ ), and then frozen at $-20^{\circ} \mathrm{C}$ for 1 day. After freeze-drying, the $\mathrm{NaCl}$ particles were leached out for 2 days in distilled water. To enhance hydrophilicity for the coating, the nanofiber mesh was treated with $1 \mathrm{~N} \mathrm{NaOH}$ for $1 \mathrm{~h}$ and the foam scaffold was treated in $\mathrm{Ar}$ (99.9\%) plasma gas (Plasma pipette, Femtoscience, Korea) for $1 \mathrm{~min}$. After vacuum treatment overnight, the samples were immersed in the Chit@IOC nanoparticle solutions at room temperature. The thickness of coating was controllable by changing the concentration of the solution $(0.5 \sim 5 \mathrm{mg} / \mathrm{mL})$ and immersion time (5 120 min). 


\section{Characterizations of nanohybrids}

The morphological and physicochemical properties of the prepared Chit@IOC nanoparticles and their bulk nanohybrids were examined using transmission electron microscopy (JEM 3010; JEOL, Japan), field emission scanning electron microscopy (FE-SEM; JEOL and HITACHI S-3000H, Japan), atomic force microscopy (AFM; SPM-9700, SHIMADZU, Japan), and Fourier transform infrared spectroscopy (FTIR; JASCO 470 PLUS, Easton, USA). Under wet conditions, the $\zeta$-potential of the nanoparticles was measured by a Zeta Sizer (ZS90, Malvern, UK). For TEM analysis, the samples were dispersed in DW, and a drop of the nanoparticles dispersion was deposited on a copper grid and air-dried. For FE-SEM analysis, the samples were sputter-coated with $\sim 10 \mathrm{~nm}$ of Pt. Infrared spectrum was recorded from samples in the solid phase in the range of 400-4000 $\mathrm{cm}^{-1}$ at a resolution of $4 \mathrm{~cm}^{-1}$. Thermogravimetric analysis (TGA) was carried out using $4 \mathrm{mg}$ of sample at a heating rate of $10{ }^{\circ} \mathrm{C} / \mathrm{min}$ at temperatures ranging from 25 to $900{ }^{\circ} \mathrm{C}$ in air. X-ray photoelectron spectrum was recorded using a PHI 5800 ESCA system (XPS; Thermo Fisher Scientific, USA) with a monochromatized aluminum $\mathrm{K}$ anode $(350 \mathrm{~W}, 25 \mathrm{~mA}$ ). The nanoparticle phase was identified by X-ray diffraction (XRD; Ultima IV, Rigaku, Japan). For the ion release test, the samples were soaked in PBS $(50 \mathrm{mg})$ and the supernatants were gathered for the analysis by inductively coupled plasma-atomic emission spectrometry (ICP-AES; OPTIMA 4300DV).

\section{Static and dynamic mechanical tests}

Tensile mechanical properties of the nanohybrids were tested under dry and wet conditions. Specimens were prepared in a thin membrane form (dimension of $10 \mathrm{~mm} \times 5 \mathrm{~mm} \times 0.21 \pm 0.016 \mathrm{~mm}$ ). The thickness of the samples was measured using a Vernier caliper at a resolution of $0.01 \mathrm{~mm}$. A static tensile load was applied to each sample. Based on the stress-strain curves recorded, the elastic modulus, maximum tensile strength, and elongation rate were calculated. Six samples were tested and the average values were recorded. A cyclic tensile load was also applied to each sample with $10 \%$ of elongation and for 30 cycles.

\section{Design of nanohybrids for multiple drug delivery}

Nanohybrids were designed to load multiple drugs. First, paclitaxel (PTX, Samyang Biopharm) and doxorubicin (DOX, hydrochloride form, TCI) were chosen for dual-drug loading. For the loading of PTX, the acidic Chit/HA solutions were homogenized in a PTX-containing solution. The solution was slowly neutralized by the addition of $1 \mathrm{M}$ ammonia solution and then dialyzed against distilled water for 2 days. The obtained aqueous Chit@HA solutions were transferred to Teflon molds, and the solvent was allowed to evaporate under ambient conditions. The obtained PTX-loaded Chit@HA nanohybrids were then fixed with 0.1 wt\% glutaraldehyde solution with the DOX drug in $0.1 \mathrm{wt} \%$ gelatin solution (30 min, $\mathrm{pH}$ 6). To remove any remnant impurities, the samples were soaked in distilled water while shaking gently overnight.

Next, triple drug delivery system was designed with Phenamil (Phe), FGF2 and dexamethasone (Dex). For this, BGn was used as a core inorganic particle. Phe (Sigma) was first homogenized with BGn in ethanol to load in the mesopores of BGn, then, the acidic Chit/BGn solution was homogenized with Phe-loaded BGn, and the supernatant was removed by centrifugation at $10,000 \mathrm{rpm}$ for $5 \mathrm{~min}$. The acidic solution was homogenized 
with another molecule FGF2 (GFP-conjugated, Sigma), which was slowly neutralized by the addition of $1 \mathrm{M}$ ammonia solution and then dialyzed against distilled water for 2 days. The obtained dual-drug (Phe and FGF2) loaded Chit@BGn solution was transferred to a Teflon mold, and the solvent was allowed to evaporate under ambient condition. The obtained nanohybrids were then fixed with $0.1 \mathrm{wt} \%$ glutaraldehyde solution containing a third molecule Dex (21-phosphate disodium form, Sigma) in $0.1 \mathrm{wt} \%$ gelatin solution (30 min, $\mathrm{pH}$ 6). To remove any remnant impurities, the samples were soaked in distilled water while shaking gently overnight. For the case of Phe-loaded Chit@ MSN nanohybrids, various compositions (with varying Chit shell thickness) were used to investigate the shell-thickness-dependent release of Phe.

\section{Analyses of drug quantity}

The amounts of drug (or dye) loaded and released from specimen $\left(1.25 \mathrm{~cm}^{2} / \mathrm{mL}\right)$ were measured by HPLC (LC20A Series, Shimadzu, Kyoto, Japan) using a stainless steel ET-RP1 4D (Shodex, Tokyo, Japan) analytical column with a diameter of $4 \mu \mathrm{m}$ and a size of $4.6 \mathrm{~mm} \times 150 \mathrm{~mm}$, and UV-vis spectrophotometry (Evolution 300, Thermo Fisher, USA). HPLC separations were conducted at a flow rate of $1 \mathrm{~mL} / \mathrm{min}$ using acetonitrile/water $(50 / 50(\mathrm{v} / \mathrm{v}))$ as the mobile phase, and the concentration of PTX was monitored at a wavelength of $210 \mathrm{~nm}$. UV-vis spectroscopy was used to determine the concentration of Dex, DOX, and Phe at $243 \mathrm{~nm}, 480 \mathrm{~nm}$ in PBS and at $368 \mathrm{~nm}$ in DMSO, respectively. Fluorescence spectroscopy was used to determine the concentration of FGF2 (by means of GFP intensity) at $538 \mathrm{~nm}$ in ethanol. Briefly, all the obtained drug (or dye)-related samples were powdered by freeze drying and then dissolved into the appropriate solvent. The prepared solutions were transferred to sample vials using syringe filters (PTFE membrane, $0.20 \mu \mathrm{m}$ ). The concentration of drug (or dye) was determined based on the calibration curve (see the Supporting Information).

\section{Mesenchymal stem cell adhesion and proliferation}

Rat mesenchymal stem cells (MSCs) were gathered from bone marrow of 5-week-old male rats in the femur and tibia according to the protocol reported previously[60]. The MSCs were used up to passage 5. A total of 80,000 cells were seeded in each specimen made to fit to each well of 24-well plates, and then cultured (from $4 \mathrm{~h}$ to 7 days). After $4 \mathrm{~h}$ of initial attachment, non-adherent cells were removed prior to further incubation. The cells were cultured in Dulbecco's modified Eagle medium (DMEM) containing 10\% fetal bovine serum (FBS, Gibco, Waltham, MA, USA) and $1 \% \mathrm{P} / \mathrm{S}$ (Gibco) in a humidified atmosphere of $37^{\circ} \mathrm{C} / 5 \% \mathrm{CO}_{2}$. To quantitate the initial cell attachment level (at 4 and $24 \mathrm{~h}$ ), the samples ( $\mathrm{n}=8$ ) were fixed with $4 \%$ PFA and then stained with DAPI for nuclei (in blue, Thermo Fisher, USA) and phalloidin for $\alpha$-actin (in red, Thermo Fisher) according to the manufacturer's protocol, and the digital images taken by fluorescence microscopy (CelenaS, Logos Biosystems, Anyang-si, Gyeonggi-do, South Korea) were quantified by ImageJ (NIH, USA, ver. 1.52a, $\mathrm{n}=8)$.

The MSC proliferation level was examined by an MTS assay (Promega, Madison, WI, USA). Briefly, for each cultured sample ( $\mathrm{n}=5$ ), $20 \mu \mathrm{L}$ of MTS solution was added to $200 \mu \mathrm{L}$ of supplemented culture medium, and cells were incubated at $37^{\circ} \mathrm{C}$ for $3 \mathrm{~h}$. The absorbance (at $490 \mathrm{~nm}$ ) was measured using a microplate reader (SpectraMax M2e, Molecular Devices). 


\section{Quantitative PCR array}

A total of 800,000 cells were seeded in each specimen for $2 \mathrm{~h}$. A total RNA was extracted from the adherent MSCs using Ribospin (GeneAll, Korea) and reverse transcribed into cDNA using a kit (AccuPower® RocketscriptTM Cycle RT Premix, BIONEER, Korea). Primers used for PCR amplification for the qPCR array were reported in BIONEER (www.bioneer.co.kr): integrin $\alpha 1, \alpha 2, \alpha 3, \alpha 4, \alpha 5, \alpha v, \beta 1, \beta 2, \beta 3$, actin, fibronectin, talin, protein tyrosine kinase, lamin a/c, vinculin, sun1, nesprin1, nesprin2, and GAPDH. The expression levels of the target genes were calculated relative to GAPDH expression, and expressed by the $2^{-\Delta \Delta \mathrm{Ct}}$ method $^{[61]}$. The real-time PCR from $1 \mu \mathrm{g}$ of reverse-transcribed total RNA was performed with the Exicycler TM 96 Real-Time Quantitative Thermal Block (BIONEER) according to the previously reported procedure[62]. All reactions were performed under the following conditions: $10 \mathrm{~min}$ at $95^{\circ} \mathrm{C} ; 40$ cycles of $5 \mathrm{~s}$ at $95^{\circ} \mathrm{C}, 25 \mathrm{~s}$ at $58^{\circ} \mathrm{C}, 30 \mathrm{~s}$ at $72^{\circ} \mathrm{C}$, and $5 \mathrm{~s}$ at $65^{\circ} \mathrm{C}$. All reactions were performed in triplicate $(\mathrm{n}=3)$.

\section{Expression of osteogenic genes}

10,000 MSCs were initially seeded in each well of 24 well-plates prior to inducing differentiation. After 14 days of osteogenic induction ( $\alpha$-MEM supplemented with 10\% FBS, 1\% P/S, $10 \mathrm{mM} \beta$-glycerophosphate, 50 $\mu \mathrm{g} / \mathrm{mL}$ ascorbic acid, and $10 \mathrm{nM}$ dexamethasone), the expression of alkaline phosphatase (ALP) and osteocalcin $(\mathrm{OCN})$ were quantitatively analyzed (normalized by growth medium-cultured group) by qPCR. In brief, the first strand cDNA was synthesized from the total RNA $(1 \mu \mathrm{g})$ mixed with $1 \mu \mathrm{g}$ oligo DT and an Accupower RT premix (K-2043, Bioneer, Korea) using VeritiTM 96-well thermal cycler (9902, Applied Biosystems, USA). Then, qRT-PCR was performed using StepOnePlus real time PCR systems (Applied Biosystems) according to the manufacturer's instructions. Relative transcriptional levels were calculated by the $2^{-\Delta \Delta \mathrm{Ct}}$ method using GAPDH as the endogenous reference gene[61] $(n=3)$. Primer sequences are: ALP forward 5'-CTCTGCCGTTGTTTCTCTAT-3', reverse 5'-AGGTGCTTTGGGAATCTG-3'. GAPDH forward 5'-CAAGGATACTGAGAGCAAGAG-3' and reverse 5'-ATGGAATTGTGAGGGAGATG-3'.

\section{Alkaline phosphatase (ALP) enzymatic activity}

10,000 MSCs were initially seeded in each well of 24 well-plates prior to inducing differentiation. After culture for 7 and 14 days, the osteogenic differentiation of MSCs cultured in differentiation medium was assessed based on the ALP enzymatic activity (Invitrogen, $n=3$ ). Each sample was added to ALP reaction medium for the enzymatic reaction. The content of the product of the enzymatic reaction ( $p$-nitrophenol) was calculated from the absorbance (at $405 \mathrm{~nm}$ ). The ALP content was normalized to the total DNA quantity (Quant-iT PicoGreen Kit, Invitrogen).

\section{In vitro biological tests of nanohybrids delivering multiple drugs}

Extracts $\left(1.25 \mathrm{~cm}^{2} / \mathrm{mL}\right)$ were used to test the biological effects of nanohybrids that load triple drugs (Dex, FGF2, and Phe). Specimens (Chit, Chit@BGn70, Chit@BGn70+triple drugs) were prepared in a thin membrane form, and the extraction was carried out in a horizontal moving shaker (120 rpm) for 7 days. Specific basal medium was used to gather the extracts at hours 4 and 12, and days 2, 4, and 7, then the extracts were treated to each type of cells; 50\% extract to macrophage cell line Raw 264.7 (ATCC TIB-71) and endothelial 
cell line HUVEC (ATCC, PCS-100-010), and 25\% extract to MSC primary cells (as described in the above section). A complete medium was used for Raw264.7 (DMEM, 10\% FBS, LPS $10 \mathrm{ng} / \mathrm{mL}$ ), while vascular cell basal medium (ATCC, PCS-100-030) supplemented with an endothelial cell growth kit-VEGF (ATCC, PCS100-041) was used for HUVEC. After the collection of each medium, the basal medium of extraction was refreshed, and after $24 \mathrm{~h}$ of cell seeding in each well of 24 well-plates, the medium was replaced.

After $4 \mathrm{~h}$ of incubation, Raw 264.7 cells treated with the extract for $4 \mathrm{~h}$ was collected for qPCR analysis following the above protocol, and the cultured medium was used to investigate NO production according to the manufacturer's protocol (iNtRON, Korea) and IL1- $\beta$ gene expression. Primer sequences are: IL-1 $\beta$ forward 5'-CTGTGACTCATGGGATGATGATG-3', reverse 5'-GCCTGTAGTGCAGTTGTCTAAT-3'; and GAPDH forward 5'-GAAACCTGCCAAGTATGATG-3', reverse 5'-GGAGTTGCTGTTGAAGTC-3'.

The osteogenesis of MSCs was conducted for up to 7 days, and the $25 \%$ extract treated to MSCs was refreshed at 4, $12 \mathrm{~h}$, and 2, 4, 7 days. The ALP staining of samples was carried out at day 4 (FAST BCIP/NBT B5655, Sigma-Aldrich), and the qPCR analysis of OCN gene was performed at day 7. Primer sequence for OCN is forward 5'-GCTTCAGCTTTGGCTACT-3', reverse 5'-CGTTCCTCATCTGGACCTTAT-3'.

For the angiogenesis study, $5 \times 10^{4}$ HUVECs were cultured with 50\% extract on Matrigel (Corning, USA) coated 24 well-plates, and the tubule formation was monitored for up to $12 \mathrm{~h}$ using a Juli Stage (NanoEntek Inc., Korea). Images for the ALP staining and tubule formation were analyzed by Image J.

\section{In vivo animal model and surgery}

The in vivo experiments were approved by the Animal Care and Use Committee at Dankook University (approval \#17-011), Republic of Korea. Healthy, male, 9-10-week-old Sprague-Dawley rats were used in the studies. Two separate experiments were conducted using; i) membrane form without drug (pure Chit, Chit@BGn50, Chit@BGn70), and ii) scaffold form with drug (Chit@BGn70 and Chit@BGn70 with triple drugs). Empty defect control was used for each experiment. Samples in a membrane form were as described in the above section. For the scaffold samples, 3D printed PCL was used as the 3D framework for better analyses of the new bone formation. The 3D printing process was described in detail in our previous work[63]. On the 3D printed PCL scaffold, the Chit@BGn70 was coated following the procedures as described in the above section. Furthermore, the triple drugs (Dex, FGF2, and Phe) were also loaded onto the scaffolds. Five specimens for each group $(\mathrm{n}=5)$ were used for the in vivo experiments.

For the operation, animals were anesthetized by an intramuscular treatment of a mixture of ketamine $(80 \mathrm{mg} / \mathrm{kg}$ ) and xylazine $(10 \mathrm{mg} / \mathrm{kg})$. After shaving over the cranial lesion, the surgical site was mopped with iodine and 70\% ethanol, and a linear incision in the skin was made with a surgical blade. A full-thickness flap was retracted, and the calvarium bone was exposed. Two 5-mm-diameter calvarium bone defects, one on each side of the parietal bone, were generated in each rat under cooling conditions with sterile saline using a dental hand-piece and 5-mm-diameter trephine drill (South Korea). The operation sites in animals were randomly assigned to experimental groups before the implantation. After the implantation, the subcutaneous tissues and periosteum were closed with absorbable sutures (4-0 Vicryl@, Ethicon, Germany), and the skin was sutured 
with non-absorbable sutures (4-0 Prolene, Ethicon, Germany). The animals were monitored daily for clinical signs indicative of infection, inflammation, or any adverse reaction. For the membrane experiment, animals were sacrificed at 5 weeks to investigate relatively early bone formation. On the other hand, animals implanted with the scaffolds were sacrificed at both early (4 weeks) and late time points (12 weeks) for the analyses. For the sacrifice, the animals were euthanized by $\mathrm{CO}_{2}$ inhalation, and the tissue surrounding the calvarium defect with implantation was harvested and fixed in $10 \%$ neutral buffered formalin for $24 \mathrm{~h}$ at room temperature for histological and micro-computed tomographical (micro-CT) analyses.

\section{Analyses by histology and micro-CT}

Micro-CT imaging was used as the representative technique for the analyses of bone regeneration [64]. The collected samples were subjected to micro-CT imaging (Skyscan 1176, Skyscan, Belgium) using X-ray at 65 $\mathrm{kV}$ and $385 \mu \mathrm{A}$ with an exposure time of $279 \mathrm{~ms}$ for each section $(\mu \mathrm{m})$. Images reconstructed from the scans were used to analyze hard tissue formation over the region of interest (ROI) using CTAnSkyscan software. The newly formed bone volume $\left(\mathrm{mm}^{3}\right)$ and surface area $\left(\mathrm{mm}^{2}\right)$ in the defect area were assessed. Threedimensional images were constructed and visualized by CTvol Skyscan software (ver. 2.3.2.0).

For the histological analyses, the fixed tissue samples were decalcified in RapidCal ${ }^{\text {TM }}$ solution (BBC Chemical Co., USA) for 14 days. After decalcification, the samples were dehydrated in a graded series of ethanol solutions and then embedded in paraffin. Coronal sections $(5 \mu \mathrm{m})$ of the central area of the defect site were prepared using a semi-automated rotary microtome (Leica RM2245, Leica Biosystems, Germany). After transferred to coated glass slides, the samples were made into tissue section slides and stained with hematoxylin and eosin (H\&E) for optical microscopy.

The immunohistochemical staining of tissue samples was performed to analyze the new bone area. For immunohistochemistry, anti-BSP II antibody (1:200, sc-73634, Santa Cruz Biotechnology Inc., USA), antiALP antibody (1:200, sc-23430, Santa Cruz Biotechnology Inc.), fluorescein (FITC) AffiniPure Goat AntiMouse IgG (1:200, Jackson ImmunoResearch Laboratories Inc., USA), and rhodamine (TRITC) AffiniPure Bovine Anti-Goat IgG (1:200, Jackson ImmunoResearch Laboratories Inc.) were used to quantify the bone protein markers BSP and ALP in the newly formed bone. The images were taken with an Olympus inverted microscope (IX71, Olympus, Tokyo, Japan). In the histology sections, the osteoblasts in the new bone area of each defect margin were counted. Cuboidal cells with eccentric nuclei were considered osteoblasts. The fluorescence intensity of the total new bone area was quantified by ImageJ software (https://imagej.nih.gov/ij/).

\section{Statistics}

Data are presented as the mean \pm one standard deviation (1SD). Comparisons among groups were carried out using one-way analysis of variance (ANOVA) with Bonferroni post hoc test. Statistical significance was considered at different levels $(* \mathrm{p}<0.05, * * \mathrm{p}<0.01)$.

\section{Supporting Information}


Figure S1 Figure S22

Table S1, S2

Video S1, S2

Conflict of Interest: All authors declare no conflict of interest.

Acknowledgments: This work was supported by the grants (2019R1C1C1002490, 2018R1A2B3003446, 2018K1A4A3A01064257, 2019R1A6A1A11034536, 2015K1A1A2032163), National Research Foundation, Republic of Korea.

Data availability: The raw/processed data required to reproduce these findings cannot be shared at this time due to technical or time limitations. However, they can be provided upon requested. 


\section{REFERENCES}

1. P. J. M. Smeets, K. R. Cho, R. G. E. Kempen, N. A. J. M. Sommerdijk, J. J. De Yoreo, Calcium carbonate nucleation driven by ion binding in a biomimetic matrix revealed by in situ electron microscopy. Nat. Mater. 14, 394 (2015).

2. J. Chung, A. M. Kushner, A. C. Weisman, Z. Guan, Direct correlation of singlemolecule properties with bulk mechanical performance for the biomimetic design of polymers. Nat. Mater. 13, 1055 (2014).

3. J. J. Green, J. H. Elisseeff, Mimicking biological functionality with polymers for biomedical applications. Nature 540, 386 (2016); published online Epub12/14/online (10.1038/nature21005).

4. P. Mohammadi, A. S. Aranko, C. P. Landowski, O. Ikkala, K. Jaudzems, W. Wagermaier, M. B. Linder, Biomimetic composites with enhanced toughening using silk-inspired triblock proteins and aligned nanocellulose reinforcements. Sci. Adv. 5, eaaw2541 (2019)10.1126/sciadv.aaw2541).

5. F. Barthelat, Biomimetics for next generation materials. Philos. Trans. R. Soc. A 365, 2907-2919 (2007).

6. O. A. Tertuliano, J. R. Greer, The nanocomposite nature of bone drives its strength and damage resistance. Nat. Mater. 15, 1195 (2016).

7. X. Deng, J. Hao, C. Wang, Preparation and mechanical properties of nanocomposites of poly (D, L-lactide) with Ca-deficient hydroxyapatite nanocrystals. Biomaterials 22, 2867-2873 (2001).

8. S. J. Peter, S. T. Miller, G. Zhu, A. W. Yasko, A. G. Mikos, In vivo degradation of a poly (propylene fumarate)/ $\beta$-tricalcium phosphate injectable composite scaffold. $J$. Biomed. Mater. Res. 41, 1-7 (1998).

9. H. Granel, C. Bossard, A.-M. Collignon, F. Wauquier, J. Lesieur, G. Y. Rochefort, E. Jallot, J. Lao, Y. Wittrant, Bioactive Glass/Polycaprolactone Hybrid with a Dual Cortical/Trabecular Structure for Bone Regeneration. ACS Appl. Bio. Mater. 2, 34733483 (2019).

10. J. Luo, X. Zhang, J. Ong'achwa Machuki, C. Dai, Y. Li, K. Guo, F. Gao, ThreeDimensionally N-Doped Graphene-Hydroxyapatite/Agarose as an Osteoinductive Scaffold for Enhancing Bone Regeneration. ACS Appl. Bio. Mater. 2, 299-310 (2018).

11. S. Tamburaci, B. Cecen, O. Ustun, B. U. Ergur, H. Havitcioglu, F. Tihminlioglu, Production and Characterization of a Novel Bilayer Nanocomposite Scaffold Composed of Chitosan/Si-nHap and Zein/POSS Structures for Osteochondral Tissue Regeneration. ACS Appl. Bio. Mater. 2, 1440-1455 (2019).

12. J. Juhasz, S. Best, R. Brooks, M. Kawashita, N. Miyata, T. Kokubo, T. Nakamura, W. Bonfield, Mechanical properties of glass-ceramic A-W-polyethylene composites: effect of filler content and particle size. Biomaterials 25, 949-955 (2004).

13. K. Zhang, Y. Wang, M. A. Hillmyer, L. F. Francis, Processing and properties of porous poly (L-lactide)/bioactive glass composites. Biomaterials 25, 2489-2500 (2004).

14. T. Kawai, S. Kamakura, K. Matsui, M. Fukuda, H. Takano, M. Iino, S. Ishikawa, H. Kawana, T. Soma, E. Imamura, Hideki Kizu, Aya Michibata, Izumi Asahina, Keiichiro Miura, Norifumi Nakamura,Toshiro Kibe, Osamu Suzuki, Tetsu Takahashi, Clinical study of octacalcium phosphate and collagen composite in oral 
and maxillofacial surgery. Journal of Tissue Engineering 10, 2041731419896449 (2020).

15. H. Maleki, M.-A. Shahbazi, S. Montes, S. H. Hosseini, M. R. Eskandari, S. Zaunschirm, T. Verwanger, S. Mathur, B. Milow, B. Krammer, Mechanically Strong Silica-Silk Fibroin Bioaerogel: A Hybrid Scaffold with Ordered Honeycomb Micromorphology and Multiscale Porosity for Bone Regeneration. ACS Appl. Mater. Interfaces, (2019).

16. N. Lu, Y. Lu, S. Liu, C. Jin, S. Fang, X. Zhou, Z. Li, Tailor-Engineered POSS-Based Hybrid Gels for Bone Regeneration. Biomacromolecules 20, 3485-3493 (2019).

17. Z. Wu, Z. Meng, Q. Wu, D. Zeng, Z. Guo, J. Yao, Y. Bian, Y. Gu, S. Cheng, L. Peng, Y. Zhao, Biomimetic and osteogenic 3D silk fibroin composite scaffolds with nano $\mathrm{MgO}$ and mineralized hydroxyapatite for bone regeneration. Journal of Tissue Engineering 10, 2041731420967791 (2020).

18. T. Kasaga, O. Yoshio, N. Masayuki, A. Yoshihiro, Preparation and mechanical properties of polylactide acid composites containing hydroxyapatite fibres. Biomaterials 22, 9-23 (2001).

19. A. E. Jakus, A. L. Rutz, S. W. Jordan, A. Kannan, S. M. Mitchell, C. Yun, K. D. Koube, S. C. Yoo, H. E. Whiteley, C.-P. Richter, Hyperelastic "bone": A highly versatile, growth factor-free, osteoregenerative, scalable, and surgically friendly biomaterial. Sci. Transl. Med. 8, 358ra127-358ra127 (2016).

20. K. Zhou, P. Yu, X. Shi, T. Ling, W. Zeng, A. Chen, W. Yang, Z. Zhou, Hierarchically Porous Hydroxyapatite Hybrid Scaffold Incorporated with Reduced Graphene Oxide for Rapid Bone Ingrowth and Repair. ACS Nano 13, 9595-9606 (2019).

21. T. M. Bedair, C. K. Lee, D.-S. Kim, S.-W. Baek, H. M. Bedair, H. P. Joshi, U. Y. Choi, K.-H. Park, W. Park, I. Han, D. K. Han, Magnesium hydroxide-incorporated PLGA composite attenuates inflammation and promotes BMP2-induced bone formation in spinal fusion. Journal of Tissue Engineering 10, 2041731420967591 (2020).

22. R. Ichikawa, S. Kajiyama, M. Iimura, T. Kato, Tuning the c-Axis Orientation of Calcium Phosphate Hybrid Thin Films Using Polymer Templates. Langmuir 35, 4077-4084 (2019).

23. A. S. Chahal, M. Schweikle, A.-M. Lian, J. E. Reseland, H. J. Haugen, H. Tiainen, Osteogenic potential of poly(ethylene glycol)-amorphous calcium phosphate composites on human mesenchymal stem cells. Journal of Tissue Engineering 10, 2041731420926840 (2020).

24. S. Mallakpour, M. Naghdi, Polymer/SiO2 nanocomposites: Production and applications. Prog. Mater. Sci. 97, 409-447 (2018); published online Epub2018/08/01/ (https://doi.org/10.1016/j.pmatsci.2018.04.002).

25. F. Delogu, G. Gorrasi, A. Sorrentino, Fabrication of polymer nanocomposites via ball milling: Present status and future perspectives. Prog. Mater. Sci. 86, 75-126 (2017); published online Epub2017/05/01/ (https://doi.org/10.1016/j.pmatsci.2017.01.003).

26. L. Gao, Y. Shi, W. Li, W. Ren, J. Liu, Y. Cai, Determination of organophosphate esters in water samples by mixed-mode liquid chromatography and tandem mass spectrometry. J. Sep Sci 38, 2193-2200 (2015).

27. R. Rama, A. Rout, K. Venkatesan, M. Antony, Effect of alkyl chain length of tri-nalkyl phosphate extractants on the electrochemical behaviour of U (VI) in ionic liquid medium. J. Electroanal. Chem. 771, 87-93 (2016). 
28. S. Subramaniam, Y.-H. Fang, S. Sivasubramanian, F.-H. Lin, C.-p. Lin, Hydroxyapatite-calcium sulfate-hyaluronic acid composite encapsulated with collagenase as bone substitute for alveolar bone regeneration. Biomaterials 74, 99108 (2016); published online Epub2016/01/01/ (https://doi.org/10.1016/j.biomaterials.2015.09.044).

29. C.-c. Jiang, Y.-k. Cao, G.-y. Xiao, R.-f. Zhu, Y.-p. Lu, A review on the application of inorganic nanoparticles in chemical surface coatings on metallic substrates. RSC Adv. 7, 7531-7539 (2017)10.1039/C6RA25841G).

30. A. E. Jakus, A. L. Rutz, S. W. Jordan, A. Kannan, S. M. Mitchell, C. Yun, K. D. Koube, S. C. Yoo, H. E. Whiteley, C.-P. Richter, R. D. Galiano, W. K. Hsu, S. R. Stock, E. L. Hsu, R. N. Shah, Hyperelastic "bone": A highly versatile, growth factorfree, osteoregenerative, scalable, and surgically friendly biomaterial. Sci. Transl. Med. 8, 358ra127-358ra127 (2016)10.1126/scitranslmed.aaf7704).

31. D. Zhi, H. Wang, D. Jiang, I. P. Parkin, X. Zhang, Reactive silica nanoparticles turn epoxy coating from hydrophilic to super-robust superhydrophobic. RSC Adv. 9, 12547-12554 (2019)10.1039/C8RA10046B).

32. M. J. Dalby, N. Gadegaard, R. O. Oreffo, Harnessing nanotopography and integrinmatrix interactions to influence stem cell fate. Nat. Mater. 13, 558-569 (2014).

33. M. J. Dalby, N. Gadegaard, R. Tare, A. Andar, M. O. Riehle, P. Herzyk, C. D. W. Wilkinson, R. O. C. Oreffo, The control of human mesenchymal cell differentiation using nanoscale symmetry and disorder. Nat. Mater. 6, 997-1003 (2007); published online Epub2007/12/01 (10.1038/nmat2013).

34. C. Tan, M. Arshadi, M. C. Lee, M. Godec, M. Azizi, B. Yan, H. Eskandarloo, T. W. Deisenroth, R. H. Darji, T. V. Pho, A Robust Aqueous Core-Shell-Shell Coconutlike Nanostructure for Stimuli-Responsive Delivery of Hydrophilic Cargo. ACS Nano 13, 9016-9027 (2019).

35. I. Tunc, S. Suzer, M. A. Correa-Duarte, L. M. Liz-Marzan, XPS characterization of $\mathrm{Au}$ (core)/SiO2 (shell) nanoparticles. J. Phys. Chem. A 109, 7597-7600 (2005).

36. J.-Y. Hwang, H.-S. Kim, J. H. Kim, U. S. Shin, S.-H. Lee, Carbon Nanotube Nanocomposites with Highly Enhanced Strength and Conductivity for Flexible Electric Circuits. Langmuir 31, 7844-7851 (2015); published online Epub2015/07/21 (10.1021/acs.langmuir.5b00845).

37. J. Z. Kechagia, J. Ivaska, P. Roca-Cusachs, Integrins as biomechanical sensors of the microenvironment. Nat. Rev. Mol. Cell Biol. 20, 457-473 (2019); published online Epub2019/08/01 (10.1038/s41580-019-0134-2).

38. J.-H. Lee, D.-H. Kim, H.-H. Lee, H.-W. Kim, Role of nuclear mechanosensitivity in determining cellular responses to forces and biomaterials. Biomaterials 197, 60-71 (2019); published online Epub2019/03/01/ (https://doi.org/10.1016/j.biomaterials.2019.01.010).

39. I. Banerjee, J. Zhang, T. Moore-Morris, E. Pfeiffer, K. S. Buchholz, A. Liu, K. Ouyang, M. J. Stroud, L. Gerace, S. M. Evans, A. McCulloch, J. Chen, Targeted Ablation of Nesprin 1 and Nesprin 2 from Murine Myocardium Results in Cardiomyopathy, Altered Nuclear Morphology and Inhibition of the Biomechanical Gene Response. PLoS Genet. 10, e1004114 (2014)10.1371/journal.pgen.1004114).

40. M. J. P. Biggs, M. J. Dalby, Focal adhesions in osteoneogenesis. Proceedings of the Institution of Mechanical Engineers. Part H, Journal of engineering in medicine 224, 1441-1453 (2010)10.1243/09544119JEIM775). 
41. M. Schvartzman, M. Palma, J. Sable, J. Abramson, X. Hu, M. P. Sheetz, S. J. Wind, Nanolithographic Control of the Spatial Organization of Cellular Adhesion Receptors at the Single-Molecule Level. Nano Lett. 11, 1306-1312 (2011); published online Epub2011/03/09 (10.1021/nl104378f).

42. H.-L. G. Kai-Run Zhang, Xiao-Feng Pan, Pu Zhou, Xin Xing, Rui Xu, Zhao Pan, Shuang Wang, Youming Zhu, Bin Hu, Duohong Zou, Shu-Hong, Multifunctional Bilayer Nanocomposite Guided Bone Regeneration Membrane. Matter 1, 770-781 (2019).

43. E. J. Lee, D. S. Shin, H. E. Kim, H. W. Kim, Y. H. Koh, J. H. Jang, Membrane of hybrid chitosan-silica xerogel for guided bone regeneration. Biomaterials 30, 743750 (2009); published online EpubFeb (10.1016/j.biomaterials.2008.10.025).

44. A. Turri, I. Elgali, F. Vazirisani, A. Johansson, L. Emanuelsson, C. Dahlin, P. Thomsen, O. Omar, Guided bone regeneration is promoted by the molecular events in the membrane compartment. Biomaterials 84, 167-183 (2016); published online EpubApr (10.1016/j.biomaterials.2016.01.034).

45. M. Furuya, J. Kikuta, S. Fujimori, S. Seno, H. Maeda, M. Shirazaki, M. Uenaka, H. Mizuno, Y. Iwamoto, A. Morimoto, K. Hashimoto, T. Ito, Y. Isogai, M. Kashii, T. Kaito, S. Ohba, U. I. Chung, A. C. Lichtler, K. Kikuchi, H. Matsuda, H. Yoshikawa, M. Ishii, Direct cell-cell contact between mature osteoblasts and osteoclasts dynamically controls their functions in vivo. Nature communications 9, 300 (2018); published online EpubJan 19 (10.1038/s41467-017-02541-w).

46. T. Niedermair, S. Schirner, R. Seebroker, R. H. Straub, S. Grassel, Substance P modulates bone remodeling properties of murine osteoblasts and osteoclasts. Scientific reports 8, 9199 (2018); published online EpubJun 15 (10.1038/s41598018-27432-y).

47. M. D. Gerry L. Koons, Antonios G. Mikos, Materials design for bone-tissue engineering. Nature Reviews Materials 5, 584-603 (2020).

48. M. Dang, L. Saunders, X. Niu, Y. Fan, P. X. Ma, Biomimetic delivery of signals for bone tissue engineering. Bone research 6, 25 (2018)10.1038/s41413-018-0025-8).

49. T. H. Kim, M. S. Kang, N. Mandakhbayar, A. El-Fiqi, H. W. Kim, Antiinflammatory actions of folate-functionalized bioactive ion-releasing nanoparticles imply drug-free nanotherapy of inflamed tissues. Biomaterials 207, 23-38 (2019); published online EpubJul (10.1016/j.biomaterials.2019.03.034).

50. K. D. Huebner, N. G. Shrive, C. B. Frank, Dexamethasone inhibits inflammation and cartilage damage in a new model of post-traumatic osteoarthritis. Journal of orthopaedic research : official publication of the Orthopaedic Research Society 32, 566-572 (2014); published online EpubApr (10.1002/jor.22568).

51. M. J. Webber, J. B. Matson, V. K. Tamboli, S. I. Stupp, Controlled release of dexamethasone from peptide nanofiber gels to modulate inflammatory response. Biomaterials 33, 6823-6832 (2012); published online EpubOct (10.1016/j.biomaterials.2012.06.003).

52. K. Dashnyam, G. Z. Jin, J. H. Kim, R. Perez, J. H. Jang, H. W. Kim, Promoting angiogenesis with mesoporous microcarriers through a synergistic action of delivered silicon ion and VEGF. Biomaterials 116, 145-157 (2017); published online EpubFeb (10.1016/j.biomaterials.2016.11.053).

53. A. El-Fiqi, N. Mandakhbayar, S. B. Jo, J. C. Knowles, J. H. Lee, H. W. Kim, Nanotherapeutics for regeneration of degenerated tissue infected by bacteria through the multiple delivery of bioactive ions and growth factor with 
antibacterial/angiogenic and osteogenic/odontogenic capacity. Bioact Mater 6, 123136 (2021); published online EpubJan (10.1016/j.bioactmat.2020.07.010).

54. H. Chu, J. Gao, C. W. Chen, J. Huard, Y. Wang, Injectable fibroblast growth factor2 coacervate for persistent angiogenesis. Proceedings of the National Academy of Sciences of the United States of America 108, 13444-13449 (2011); published online EpubAug 16 (10.1073/pnas.1110121108).

55. J. H. Lee, N. Mandakhbayar, A. El-Fiqi, H. W. Kim, Intracellular co-delivery of Sr ion and phenamil drug through mesoporous bioglass nanocarriers synergizes BMP signaling and tissue mineralization. Acta Biomater 60, 93-108 (2017); published online EpubSep 15 (10.1016/j.actbio.2017.07.021).

56. J. Fan, C. S. Im, Z. K. Cui, M. Guo, O. Bezouglaia, A. Fartash, J. Y. Lee, J. Nguyen, B. M. Wu, T. Aghaloo, M. Lee, Delivery of Phenamil Enhances BMP-2-Induced Osteogenic Differentiation of Adipose-Derived Stem Cells and Bone Formation in Calvarial Defects. Tissue engineering. Part A 21, 2053-2065 (2015); published online EpubJul (10.1089/ten.TEA.2014.0489).

57. S. Baek, R. K. Singh, D. Khanal, K. D. Patel, E.-J. Lee, K. W. Leong, W. Chrzanowski, H.-W. Kim, Smart multifunctional drug delivery towards anticancer therapy harmonized in mesoporous nanoparticles. Nanoscale 7, 14191-14216 (2015).

58. J.-H. Lee, M.-S. Kang, C. Mahapatra, H.-W. Kim, Effect of aminated mesoporous bioactive glass nanoparticles on the differentiation of dental pulp stem cells. PLoS One 11, e0150727 (2016).

59. R. K. Singh, G.-Z. Jin, C. Mahapatra, K. D. Patel, W. Chrzanowski, H.-W. Kim, Mesoporous Silica-Layered Biopolymer Hybrid Nanofibrous Scaffold: A Novel Nanobiomatrix Platform for Therapeutics Delivery and Bone Regeneration. ACS Appl. Mater. Interfaces 7, 8088-8098 (2015); published online Epub2015/04/22 (10.1021/acsami.5b00692).

60. H.-J. Moon, J.-H. Lee, J.-H. Kim, J. C. Knowles, Y.-B. Cho, D.-H. Shin, H.-H. Lee, H.-W. Kim, Reformulated mineral trioxide aggregate components and the assessments for use as future dental regenerative cements. J. Tissue Eng. 9, 2041731418807396-2041731418807396 (2018)10.1177/2041731418807396).

61. J.-J. Kim, A. El-Fiqi, H.-W. Kim, Synergetic Cues of Bioactive Nanoparticles and Nanofibrous Structure in Bone Scaffolds to Stimulate Osteogenesis and Angiogenesis. ACS Appl. Mater. Interfaces 9, 2059-2073 (2017); published online Epub2017/01/25 (10.1021/acsami.6b12089).

62. B. R. Choi, H. K. Kim, K. K. Soni, K. K. Karna, S. W. Lee, I. So, J. K. Park, Additive effect of oral LDD175 to tamsulosin and finasteride in a benign prostate hyperplasia rat model. Drug Des. Devel Ther 12, 1855 (2018).

63. J. E. Won, Y. S. Lee, J. H. Park, J. H. Lee, Y. S. Shin, C. H. Kim, J. C. Knowles, H. W. Kim, Hierarchical microchanneled scaffolds modulate multiple tissueregenerative processes of immune-responses, angiogenesis, and stem cell homing. Biomaterials 227, 119548 (2020); published online EpubJan (10.1016/j.biomaterials.2019.119548).

64. E. A. Fragogeorgi, M. Rouchota, M. Georgiou, M. Velez, P. Bouziotis, G. Loudos, In vivo imaging techniques for bone tissue engineering. Journal of Tissue Engineering 10, 2041731419854586 (2019) 
